\title{
PATTERNS GENERATION AND SPATIAL ENTROPY IN TWO-DIMENSIONAL LATTICE MODELS*
}

\author{
JUNG-CHAO BAN ${ }^{\dagger}$, SONG-SUN LIN ${ }^{\ddagger}$, AND YIN-HENG LIN ${ }^{\S}$
}

\begin{abstract}
Patterns generation problems in two-dimensional lattice models are studied. Let $\mathcal{S}$ be the set of $p$ symbols and $\mathbb{Z}_{2 \ell \times 2 \ell}, \ell \geq 1$, be a fixed finite square sublattice of $\mathbb{Z}^{2}$. Function $U: \mathbb{Z}_{2 \ell \times 2 \ell} \rightarrow \mathcal{S}$ is called local pattern. Given a basic set $\mathcal{B}$ of local patterns, a unique transition matrix $\mathbb{A}_{2}$ which is a $q^{2} \times q^{2}$ matrix, $q=p^{\ell^{2}}$, can be defined. The recursive formulae of higher transition matrix $\mathbb{A}_{n}$ on $\mathbb{Z}_{2 \ell \times n \ell}$ have already been derived [4]. Now $\mathbb{A}_{n}^{m}, m \geq 1$, contains all admissible patterns on $\mathbb{Z}_{(m+1) \ell \times n \ell}$ which can be generated by $\mathcal{B}$. In this paper, the connecting operator $\mathbb{C}_{m}$, which comprises all admissible patterns on $\mathbb{Z}_{(m+1) \ell \times 2 \ell}$, is carefully arranged. $\mathbb{C}_{m}$ can be used to extend $\mathbb{A}_{n}^{m}$ to $\mathbb{A}_{n+1}^{m}$ recursively for $n \geq 2$. Furthermore, the lower bound of spatial entropy $h\left(\mathbb{A}_{2}\right)$ can be derived through the diagonal part of $\mathbb{C}_{m}$. This yields a powerful method for verifying the positivity of spatial entropy which is important in examining the complexity of the set of admissible global patterns. The trace operator $\mathbb{T}_{m}$ of $\mathbb{C}_{m}$ can also be introduced. In the case of symmetric $\mathbb{A}_{2}, \mathbb{T}_{2 m}$ gives a good estimate of the upper bound on spatial entropy. Combining $\mathbb{C}_{m}$ with $\mathbb{T}_{m}$ helps to understand the patterns generation problems more systematically.
\end{abstract}

Key words. Lattice dynamical systems, Spatial entropy, Patterns generation, Connecting operator, Trace operator

AMS subject classifications. Primary 37B50; Secondary 37B40

1. Introduction. Lattices are important in scientifically modelling underlying spatial structures. Investigations in this field have covered phase transition [11], [12], [34], [35], [36], [37], [38], [45], [46], [47], [48], chemical reaction [7], [8], [24], biology $[9],[10],[21],[22],[23],[31],[32],[33]$ and image processing and pattern recognition $[16],[17],[18],[19],[20],[25]$. In the field of lattice dynamical systems (LDS) and cellular neural networks (CNN), the complexity of the set of all global patterns recently attracted substantial interest. In particular, its spatial entropy has received considerable attention [1], [2], [3], [4], [5], [13], [14], [15], [28], [29],[30], [39], [40], [41], [42], [43], [44].

The one dimensional spatial entropy $h$ can be found from an associated transition matrix $\mathbb{T}$. The spatial entropy $h$ equals $\log \rho(\mathbb{T})$, where $\rho(\mathbb{T})$ is the maximum eigenvalue of $\mathbb{T}$.

In two-dimensional situations, higher transition matrices have been discovered in [30] and developed systematically [4] by studying the patterns generation problem.

This study extends our previous work [4]. For simplicity, two symbols on $2 \times$ 2 lattice $\mathbb{Z}_{2 \times 2}$ are considered. A transition matrix in the horizontal (or vertical)

\footnotetext{
${ }^{*}$ Received October 18, 2005; accepted for publication July 25, 2006. This reserch is partially supported by the National Science Council, R.O.C.(Contract No. NSC 94-2115-M-009-002) and the National Center for Theoretical Sciences.

†The National Center for Theoretical Sciences, Hsin-Chu 300, Taiwan (jcban@math.cts.nthu. edu.tw).

${ }^{\ddagger}$ Department of Applied Mathematics, National Chiao Tung University, Hsinchu 300, Taiwan (sslin@math.nctu.edu.tw).

$\S$ Department of Applied Mathematics, National Chiao Tung University, Hsinchu 300, Taiwan (yhlin.am91g@nctu.edu.tw).
} 
direction

$$
\mathbb{A}_{2}=\left[\begin{array}{llll}
a_{11} & a_{12} & a_{13} & a_{14} \\
a_{21} & a_{22} & a_{23} & a_{24} \\
a_{31} & a_{32} & a_{33} & a_{34} \\
a_{41} & a_{42} & a_{43} & a_{44}
\end{array}\right]
$$

which is linked to a set of admissible local patterns on $\mathbb{Z}_{2 \times 2}$ is considered, where $a_{i j} \in\{0,1\}$ for $1 \leq i, j \leq 4$. The associated vertical (or horizontal) transition matrix $\mathbb{B}_{2}$ is given by

$$
\mathbb{B}_{2}=\left[\begin{array}{llll}
b_{11} & b_{12} & b_{13} & b_{14} \\
b_{21} & b_{22} & b_{23} & b_{24} \\
b_{31} & b_{32} & b_{33} & b_{34} \\
b_{41} & b_{42} & b_{43} & b_{44}
\end{array}\right]
$$

$\mathbb{A}_{2}$ and $\mathbb{B}_{2}$ are connected to each other as follows.

$$
\mathbb{A}_{2}=\left[\begin{array}{ll|ll}
b_{11} & b_{12} & b_{21} & b_{22} \\
b_{13} & b_{14} & b_{23} & b_{24} \\
\hline b_{31} & b_{32} & b_{41} & b_{42} \\
b_{33} & b_{34} & b_{43} & b_{44}
\end{array}\right]=\left[\begin{array}{ll}
A_{2 ; 1} & A_{2 ; 2} \\
A_{2 ; 3} & A_{2 ; 4}
\end{array}\right],
$$

and

$$
\mathbb{B}_{2}=\left[\begin{array}{cc|cc}
a_{11} & a_{12} & a_{21} & a_{22} \\
a_{13} & a_{14} & a_{23} & a_{24} \\
\hline a_{31} & a_{32} & a_{41} & a_{42} \\
a_{33} & a_{34} & a_{43} & a_{44}
\end{array}\right]=\left[\begin{array}{cc}
B_{2 ; 1} & B_{2 ; 2} \\
B_{2 ; 3} & B_{2 ; 4}
\end{array}\right] .
$$

Notably if $\mathbb{A}_{2}$ represents the horizontal (or vertical) transition matrix then $\mathbb{B}_{2}$ represents the vertical (or horizontal) transition matrix. Results that hold for $\mathbb{A}_{2}$ are also valid for $\mathbb{B}_{2}$. Therefore, for simplicity, only $\mathbb{A}_{2}$ is presented herein.

The recursive formulae for $n$-th order transition matrices $\mathbb{A}_{n}$ defined on $\mathbb{Z}_{2 \times n}$ were obtained [4] as follows

$$
\mathbb{A}_{n+1}=\left[\begin{array}{llll}
b_{11} A_{n ; 1} & b_{12} A_{n ; 2} & b_{21} A_{n ; 1} & b_{22} A_{n ; 2} \\
b_{13} A_{n ; 3} & b_{14} A_{n ; 4} & b_{23} A_{n ; 3} & b_{24} A_{n ; 4} \\
b_{31} A_{n ; 1} & b_{32} A_{n ; 2} & b_{41} A_{n ; 1} & b_{42} A_{n ; 2} \\
b_{33} A_{n ; 3} & b_{34} A_{n ; 4} & b_{43} A_{n ; 3} & b_{44} A_{n ; 4}
\end{array}\right]
$$

whenever

$$
\mathbb{A}_{n}=\left[\begin{array}{cc}
A_{n ; 1} & A_{n ; 2} \\
A_{n ; 3} & A_{n ; 4}
\end{array}\right]
$$

for $n \geq 2$, or equivalently,

$$
A_{n+1 ; \alpha}=\left[\begin{array}{cc}
b_{\alpha 1} A_{n ; 1} & b_{\alpha 2} A_{n ; 2} \\
b_{\alpha 3} A_{n ; 3} & b_{\alpha 4} A_{n ; 4}
\end{array}\right]
$$


for $\alpha \in\{1,2,3,4\}$. The number of all admissible patterns defined on $\mathbb{Z}_{m \times n}$ which can be generated from $\mathbb{A}_{2}$ is now defined by

$$
\begin{aligned}
\Gamma_{m, n}\left(\mathbb{A}_{2}\right) & =\left|\mathbb{A}_{n}^{m-1}\right| \\
& =\text { the summation of all entries in } 2^{n} \times 2^{n} \text { matrix } \mathbb{A}_{n}^{m-1} .
\end{aligned}
$$

The spatial entropy $h\left(\mathbb{A}_{2}\right)$ is defined as

$$
h\left(\mathbb{A}_{2}\right)=\lim _{m, n \rightarrow \infty} \frac{1}{m n} \log \Gamma_{m, n}\left(\mathbb{A}_{2}\right)=\lim _{m, n \rightarrow \infty} \frac{1}{m n} \log \left|\mathbb{A}_{n}^{m-1}\right| .
$$

The existence of the limit (1.9) has been shown in [4], [15], [30]. When $h\left(\mathbb{A}_{2}\right)>0$, the number of admissible patterns grows exponentially with the lattice size $m \times n$. In this situation, spatial chaos arises. When $h\left(\mathbb{A}_{2}\right)=0$, pattern formation occurs.

To compute the double limit in (1.9), $n \geq 2$ can be fixed initially and $m$ allowed to tend to infinite [30] and [4]; then the Perron-Frobenius theorem is applied;

$$
\lim _{m \rightarrow \infty} \frac{1}{m} \log \left|\mathbb{A}_{n}^{m-1}\right|=\log \rho\left(\mathbb{A}_{n}\right),
$$

which implies

$$
h\left(\mathbb{A}_{2}\right)=\lim _{n \rightarrow \infty} \frac{1}{n} \log \rho\left(\mathbb{A}_{n}\right),
$$

where $\rho(M)$ is the maximum eigenvalue of matrix $M$. $\mathbb{A}_{n}$ is a $2^{n} \times 2^{n}$ matrix, so computing $\rho\left(\mathbb{A}_{n}\right)$ is usually quite difficult when $n$ is larger. Moreover, (1.11) does not produce any error estimation in the estimated sequence $\frac{1}{n} \log \rho\left(\mathbb{A}_{n}\right)$ and its limit $h\left(\mathbb{A}_{2}\right)$. This causes a serious problem in computing the entropy. However, for a class of $\mathbb{A}_{2}$, the recursive formulae for $\rho\left(\mathbb{A}_{n}\right)$ can be discovered, along with a limiting equation to $\rho^{*}=\exp \left(h\left(\mathbb{A}_{2}\right)\right)$, as in [4].

This study takes a different approach to resolve these difficulties. Previously, the double limit (1.9) was initially examined by taking the $m$-limit firstly as in (1.10). Now, for each fixed $m \geq 2$, the $n$-limit in (1.9) is studied. Therefore, the limit

$$
\lim _{n \rightarrow \infty} \frac{1}{n} \log \left|\mathbb{A}_{n}^{m-1}\right|
$$

is considered. Write

$$
\mathbb{A}_{n}^{m}=\left[\begin{array}{ll}
A_{m, n ; 1} & A_{m, n ; 2} \\
A_{m, n ; 3} & A_{m, n ; 4}
\end{array}\right]
$$

The investigation of (1.12) would be simpler if a recursive formula such as (1.7) could be found for $A_{m, n ; \alpha}$. The first task in this study is to solve this problem. For matrix multiplication, the indices of $A_{n ; \alpha}, \alpha \in\{1,2,3,4\}$ are conveniently expressed as

$$
\mathbb{A}_{n}=\left[\begin{array}{cc}
A_{n ; 11} & A_{n ; 12} \\
A_{n ; 21} & A_{n ; 22}
\end{array}\right] \text {. }
$$

Then

$$
A_{m, n ; \alpha}=\sum_{k=1}^{2^{m-1}} A_{m, n ; \alpha}^{(k)}
$$


where

$$
\begin{aligned}
A_{m, n ; \alpha}^{(k)} & =A_{n ; j_{1} j_{2}} A_{n ; j_{2} j_{3}} \cdots A_{n ; j_{m} j_{m+1}}, \\
k & =1+\sum_{i=2}^{m} 2^{m-i}\left(j_{i}-1\right),
\end{aligned}
$$

and

$$
\alpha=2\left(j_{1}-1\right)+j_{m+1}
$$

$A_{m, n ; \alpha}^{(k)}$ in (1.16) is called an elementary pattern of order $(m, n)$, and is a fundamental element in constructing $A_{m, n ; \alpha}$ in (1.15). Notably the elementary patterns are in lexicographic order, according to (1.17). As in [4], the following $m$-th order ordering matrix.

$$
\mathbb{X}_{m, n}=\left[\begin{array}{ll}
X_{m, n ; 1} & X_{m, n ; 2} \\
X_{m, n ; 3} & X_{m, n ; 4}
\end{array}\right]
$$

is represented to record systematically these elementary patterns, where

$$
X_{m, n ; \alpha}=\left(A_{m, n ; \alpha}^{(k)}\right)_{1 \leq k \leq 2^{m-1}}^{t}
$$

is a $2^{m-1}$ column vector.

The first main result of this study is to introduce the connecting operator $\mathbb{C}_{m}$, and to use it to derive a recursive formula like (1.7) for $A_{m, n ; \alpha}^{(k)}$. Indeed,

$$
\begin{aligned}
\mathbb{C}_{m} & =\left[\begin{array}{llll}
C_{m ; 11} & C_{m ; 12} & C_{m ; 13} & C_{m ; 14} \\
C_{m ; 21} & C_{m ; 22} & C_{m ; 23} & C_{m ; 24} \\
C_{m ; 31} & C_{m ; 32} & C_{m ; 33} & C_{m ; 34} \\
C_{m ; 41} & C_{m ; 42} & C_{m ; 43} & C_{m ; 44}
\end{array}\right] \\
& =\left[\begin{array}{llll}
S_{m ; 11} & S_{m ; 12} & S_{m ; 21} & S_{m ; 22} \\
S_{m ; 13} & S_{m ; 14} & S_{m ; 23} & S_{m ; 24} \\
S_{m ; 31} & S_{m ; 32} & S_{m ; 41} & S_{m ; 42} \\
S_{m ; 33} & S_{m ; 34} & S_{m ; 43} & S_{m ; 44}
\end{array}\right],
\end{aligned}
$$

where

$$
\begin{aligned}
C_{m ; i j} & =\left(\left[\begin{array}{ll}
a_{i 1} & a_{i 2} \\
a_{i 3} & a_{i 4}
\end{array}\right] \circ\left(\hat{\otimes}\left[\begin{array}{cc}
B_{2 ; 1} & B_{2 ; 2} \\
B_{2 ; 3} & B_{2 ; 4}
\end{array}\right]^{m-2}\right)_{2 \times 2}\right)_{2^{m-1} \times 2^{m-1}} \\
& \circ\left(E_{2^{m-2} \times 2^{m-2}} \otimes\left[\begin{array}{ll}
a_{1 j} & a_{2 j} \\
a_{3 j} & a_{4 j}
\end{array}\right]\right)_{2^{m-1} \times 2^{m-1}}
\end{aligned}
$$

is a $2^{m-1} \times 2^{m-1}$ matrix where $E_{k \times k}$ is the $k \times k$ full matrix; $\otimes$ denotes the Kronecker product, $\circ$ denotes the Hadamard product and the product $\hat{\otimes}$ which involves both the Kronecker product and the Hadamard product, as stipulated by Definition 2.2. 
In Theorem 2.4, $C_{m ; i j}$ is shown to be $a_{i_{1} i_{2}} a_{i_{2} i_{3}} \cdots a_{i_{m} i_{m+1}}$, with $i_{1}=i$ and $i_{m+1}=$ $j$. Therefore, all admissible paths of $\mathbb{A}_{2}$ from $i$ to $j$ with length $m$ are arranged systematically in matrix $C_{m ; i j}$. Now, the recursive formula is

$$
A_{m, n+1 ; \alpha}^{(k)}=\left[\begin{array}{ll}
\sum_{l=1}^{2^{m-1}}\left(S_{m ; \alpha 1}\right)_{k l} A_{m, n ; 1}^{(l)} & \sum_{l=1}^{2^{m-1}}\left(S_{m ; \alpha 2}\right)_{k l} A_{m, n ; 2}^{(l)} \\
\sum_{l=1}^{2^{m-1}}\left(S_{m ; \alpha 3}\right)_{k l} A_{m, n ; 3}^{(l)} & \sum_{l=1}^{2^{m-1}}\left(S_{m ; \alpha 4}\right)_{k l} A_{m, n ; 4}^{(l)}
\end{array}\right],
$$

for $m \geq 2, n \geq 2,1 \leq k \leq 2^{m-1}$ and $1 \leq \alpha \leq 4$. (1.24) is the generalization of (1.7).

The recursive formula (1.24) immediately yields a lower bound on entropy. Indeed, for any positive integer $K$ and diagonal periodic cycle $\beta_{1} \beta_{2} \cdots \beta_{K} \beta_{K+1}$, where $\beta_{j} \in\{1,4\}$ and $\beta_{K+1}=\beta_{1}$,

$$
h\left(\mathbb{A}_{2}\right) \geq \frac{1}{m K} \log \rho\left(S_{m ; \beta_{1} \beta_{2}} S_{m ; \beta_{2} \beta_{3}} \cdots S_{m ; \beta_{K} \beta_{K+1}}\right) .
$$

Equation (1.25) implies $h\left(\mathbb{A}_{2}\right)>0$, if a diagonal periodic cycle of $\beta_{1} \beta_{2} \cdots \beta_{K} \beta_{1}$ applies, with a maximum eigenvalue of $S_{m ; \beta_{1} \beta_{2}} \cdots S_{m ; \beta_{K} \beta_{1}}$ that greater than one. This method powerfully yields the positivity of spatial entropy, which is hard in examining the complexity of patterns generation problems.

However, the subadditivity of $\Gamma_{m, n}\left(\mathbb{A}_{2}\right)$ is known to imply

$$
h\left(\mathbb{A}_{2}\right) \leq \frac{1}{m n} \log \Gamma_{m, n}\left(\mathbb{A}_{2}\right)
$$

as in [15]. Consequently, (1.8), (1.10) and (1.26) indicate an upper bound of entropy as

$$
h\left(\mathbb{A}_{2}\right) \leq \frac{1}{n} \log \rho\left(\mathbb{A}_{n}\right)
$$

for any $n \geq 2$.

However, the Perron-Frobenius theorem also implies

$$
\limsup _{m \rightarrow \infty} \frac{1}{m} \log \operatorname{tr}\left(\mathbb{A}_{n}^{m-1}\right)=\log \rho\left(\mathbb{A}_{n}\right),
$$

where $\operatorname{tr}(M)$ denotes the trace of matrix $M$ [26], [27]. Therefore, (1.28) implies

$$
h\left(\mathbb{A}_{2}\right)=\limsup _{m, n \rightarrow \infty} \frac{1}{m n} \log \operatorname{tr}\left(\mathbb{A}_{n}^{m-1}\right) .
$$

In studying the double-limit of (1.29), for each fixed $m \geq 2$, the $n$-limit in (1.29)

$$
\limsup _{n \rightarrow \infty} \frac{1}{n} \log \operatorname{tr}\left(\mathbb{A}_{n}^{m-1}\right)
$$

is first considered. (1.30) can be studied by introducing the following trace operator

$$
\mathbb{T}_{m}=\left[\begin{array}{ll}
C_{m ; 11} & C_{m ; 22} \\
C_{m ; 33} & C_{m ; 44}
\end{array}\right] .
$$


Then, a recursive formula for $\operatorname{tr}\left(\mathbb{A}_{n}^{m}\right)$ can be verified

$$
\operatorname{tr}\left(\mathbb{A}_{n}^{m}\right)=\left|\mathbb{T}_{m}^{n-2}\left(\begin{array}{c}
\operatorname{tr} X_{m, 2 ; 1} \\
\operatorname{tr} X_{m, 2 ; 4}
\end{array}\right)\right|
$$

for $n \geq 2$, where $\operatorname{tr}\left(X_{m, n ; \alpha}\right)=\left(\operatorname{tr} A_{m, n ; \alpha}^{(k)}\right)_{1 \leq k \leq 2^{m-1}}^{t}$ and $|v|=\sum_{j=1}^{l} v_{j}$ for vector $v=$ $\left(v_{1}, \cdots, v_{l}\right)^{t}$. Consequently, (1.29) and (1.32) yield

$$
h\left(\mathbb{A}_{2}\right) \geq \limsup _{m \rightarrow \infty} \frac{1}{m} \log \rho\left(\mathbb{T}_{m}\right) .
$$

Notably, for a large class of $\mathbb{A}_{2}$, the limit sup in (1.28), (1.29), (1.30) and (1.33) can be replaced by limit. See section 3 for details.

Now, (1.33) can be applied to find the upper bounds of entropy. For example, when $\mathbb{A}_{2}$ is symmetric,

$$
h\left(\mathbb{A}_{2}\right) \leq \frac{1}{2 m} \log \rho\left(\mathbb{T}_{2 m}\right)
$$

for any $m \geq 1$. Since

$$
\mathbb{T}_{n} \leq \mathbb{B}_{n}
$$

can be shown for any $n \geq 2$. Generally, (1.33) and (1.34) yield better approximation than (1.11) and (1.27).

In summary, this study yields lower-bound estimates of entropy like (1.25) by introducing connecting operators $\mathbb{C}_{m}$, and upper-bound estimates of entropy like (1.34) by introducing trace operators $\mathbb{T}_{m}$. This approach accurately and effectively yields the spatial entropy.

The rest of this paper is organized as follows. Section 2 derives the connecting operator $\mathbb{C}_{m}$ which can recursively reduce higher order elementary patterns to patterns of lower order. Then, the lower-bound of spatial entropy can be found by computing the maximum eigenvalues of the diagonal periodic cycles of sequence $S_{m ; \alpha \beta}$. Section 3 addresses the trace operator $\mathbb{T}_{m}$ of $\mathbb{C}_{m}$. The entropy can be calculated by computing the maximum eigenvalues of $\mathbb{T}_{m}$. When $\mathbb{A}_{2}$ is symmetric, the upper-bounds of entropy are also found. Section 4 briefly discusses the theory for many symbols on larger lattices.

\section{Connecting Operators.}

2.1. Connecting operators and ordering matrices. This section derives connecting operators and investigates their properties. For clarity, two symbols on $2 \times 2$ lattice $\mathbb{Z}_{2 \times 2}$ are examined first. Section 4 addresses more general situations.

Let $\mathbb{A}_{2}$ and $\mathbb{B}_{2}$ be defined as in (1.1) (1.4). The column matrices $\widetilde{\mathbb{A}_{2}}$ and $\widetilde{\mathbb{B}_{2}}$ of $\mathbb{A}_{2}$ and $\mathbb{B}_{2}$ are defined by

$$
\widetilde{\mathbb{A}_{2}}=\left[\begin{array}{cc|cc}
a_{11} & a_{21} & a_{12} & a_{22} \\
a_{31} & a_{41} & a_{32} & a_{42} \\
\hline a_{13} & a_{23} & a_{14} & a_{24} \\
a_{33} & a_{43} & a_{34} & a_{44}
\end{array}\right]=\left[\begin{array}{cc}
\tilde{A}_{2 ; 1} & \tilde{A}_{2 ; 2} \\
\tilde{A}_{2 ; 3} & \tilde{A}_{2 ; 4}
\end{array}\right]
$$


and

$$
\widetilde{\mathbb{B}_{2}}=\left[\begin{array}{cc|cc}
b_{11} & b_{21} & b_{12} & b_{22} \\
b_{31} & b_{41} & b_{32} & b_{42} \\
\hline b_{13} & b_{23} & b_{14} & b_{24} \\
b_{33} & b_{43} & b_{34} & b_{44}
\end{array}\right]=\left[\begin{array}{cc}
\tilde{B}_{2 ; 1} & \tilde{B}_{2 ; 2} \\
\tilde{B}_{2 ; 3} & \tilde{B}_{2 ; 4}
\end{array}\right],
$$

respectively.

For matrices of higher order $n \geq 2, \mathbb{A}_{n}, \mathbb{A}_{n+1}$ and $A_{n+1 ; \alpha}$ are defined as in $(1.5) \sim(1.7)$.

For matrix multiplication, the indices of $A_{n ; \alpha}$ are conveniently expressed as

$$
\mathbb{A}_{n}=\left[\begin{array}{cc}
A_{n ; 11} & A_{n ; 12} \\
A_{n ; 21} & A_{n ; 22}
\end{array}\right]
$$

Clearly, $A_{n ; \alpha}=A_{n ; j_{1} j_{2}}$, where

$$
\alpha=\alpha\left(j_{1}, j_{2}\right)=2\left(j_{1}-1\right)+j_{2}
$$

For $m \geq 2$, the elementary pattern in the entries of $\mathbb{A}_{n}^{m}$ is represented by

$$
A_{n ; j_{1} j_{2}} A_{n ; j_{2} j_{3}} \cdots A_{n ; j_{m} j_{m+1}}
$$

where $j_{s} \in\{1,2\}$. A lexicographic order for multiple indices

$$
J_{m+1}=\left(j_{1} j_{2} \cdots j_{m} j_{m+1}\right)
$$

is introduced, using

$$
\chi\left(J_{m+1}\right)=1+\sum_{s=2}^{m} 2^{m-s}\left(j_{s}-1\right)
$$

Now,

$$
A_{m, n ; \alpha}^{(k)}=A_{n ; j_{1} j_{2}} A_{n ; j_{2} j_{3}} \cdots A_{n ; j_{m} j_{m+1}},
$$

where

$$
\alpha=\alpha\left(j_{1}, j_{m+1}\right)=2\left(j_{1}-1\right)+j_{m+1}
$$

and

$$
k=\chi\left(J_{m+1}\right)
$$

is given in (2.5). Notably, (2.5) and (2.8) do not involve $j_{m+1}$ but (2.7)does.

Therefore, $\mathbb{A}_{n}^{m}$ can be expressed as

$$
\mathbb{A}_{n}^{m}=\left[\begin{array}{cc}
A_{m, n ; 1} & A_{m, n ; 2} \\
A_{m, n ; 3} & A_{m, n ; 4}
\end{array}\right],
$$

where

$$
A_{m, n ; \alpha}=\sum_{k=1}^{2^{m-1}} A_{m, n ; \alpha}^{(k)}
$$


Furthermore,

$$
X_{m, n ; \alpha}=\left(A_{m, n ; \alpha}^{(k)}\right)_{1 \leq k \leq 2^{m-1}}^{t} .
$$

$1 \leq k \leq 2^{m-1}, X_{m, n ; \alpha}$ is a $2^{m-1}$ column-vector that consists of all elementary patterns in $A_{m, n ; \alpha}$. The ordering matrix $\mathbb{X}_{m, n}$ of $\mathbb{A}_{n}^{m}$ is now defined by

$$
\mathbb{X}_{m, n}=\left[\begin{array}{cc}
X_{m, n ; 1} & X_{m, n ; 2} \\
X_{m, n ; 3} & X_{m, n ; 4}
\end{array}\right]
$$

The ordering matrix $\mathbb{X}_{m, n}$ allows the elementary patterns to be tracked during the reduction from $\mathbb{A}_{n+1}^{m}$ to $\mathbb{A}_{n}^{m}$. This careful book-keeping provides a systematic way to generate the admissible patterns and later, lower-bound estimates of spatial entropy.

The following simplest example is studied first to illustrate the above concept.

Example 2.1. For $m=2$, the following can easily be verified;

$$
\mathbb{A}_{n}^{2}=\left[\begin{array}{cc}
A_{n ; 11}^{2}+A_{n ; 12} A_{n ; 21} & A_{n ; 11} A_{n ; 12}+A_{n ; 12} A_{n ; 22} \\
A_{n ; 21} A_{n ; 11}+A_{n ; 22} A_{n ; 21} & A_{n ; 21} A_{n ; 12}+A_{n ; 22}^{2}
\end{array}\right],
$$

and

$$
\left.\begin{array}{ll}
A_{2, n ; 1}^{(1)}=A_{n ; 11}^{2}, & A_{2, n ; 1}^{(2)}=A_{n ; 12} A_{n ; 21}, \\
A_{2, n ; 2}^{(1)}=A_{n ; 11} A_{n ; 12}, & A_{2, n ; 2}^{(2)}=A_{n ; 12} A_{n ; 22}, \\
A_{2, n ; 3}^{(1)}=A_{n ; 21} A_{n ; 11}, & A_{2, n ; 3}^{(2)}=A_{n ; 22} A_{n ; 21}, \\
A_{2, n ; 4}^{(1)}=A_{n ; 21} A_{n ; 12}, & A_{2, n ; 4}^{(2)}=A_{n ; 22}^{2} .
\end{array}\right\} .
$$

Therefore,

$$
\left.\begin{array}{l}
X_{2, n ; 1}=\left[\begin{array}{c}
A_{n ; 11}^{2} \\
A_{n ; 12} A_{n ; 21}
\end{array}\right], \quad X_{2, n ; 2}=\left[\begin{array}{c}
A_{n ; 11} A_{n, 12} \\
A_{n ; 12} A_{n ; 22}
\end{array}\right], \\
X_{2, n ; 3}=\left[\begin{array}{c}
A_{n ; 21} A_{n ; 11} \\
A_{n ; 22} A_{n ; 21}
\end{array}\right], \quad X_{2, n ; 4}=\left[\begin{array}{c}
A_{n ; 21} A_{n, 12} \\
A_{n ; 22}^{2}
\end{array}\right] .
\end{array}\right\} .
$$

Applying (1.7), and by a straightforward computation,

$$
X_{2, n+1 ; 1}=\left[\begin{array}{c}
A_{n+1 ; 11}^{2} \\
A_{n+1 ; 12} A_{n+1 ; 21}
\end{array}\right]
$$

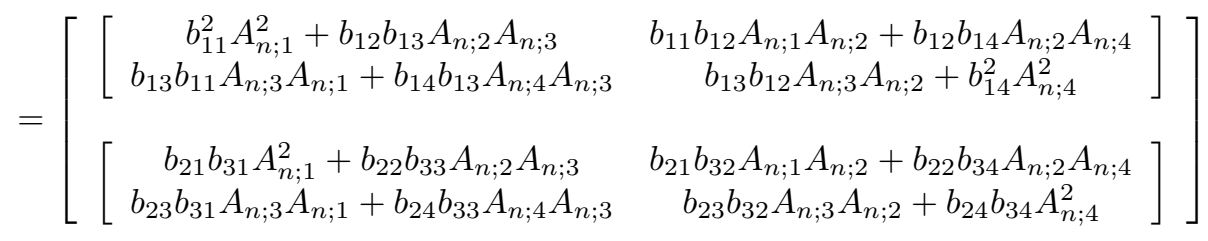

Clearly, the $j_{1} j_{2}$ entries of $A_{n+1 ; 11}^{2}$ and $A_{n+1 ; 12} A_{n+1 ; 21}$ in (2.16) consist of entries of $X_{2, n ; \alpha}$ in (2.14) with $\alpha=\alpha\left(j_{1}, j_{2}\right)$ in (2.4). Moreover, the terms in (2.16) can be 
rearranged in terms of $X_{2, n ; \alpha}$ by exchanging the second row in the first matrix with the first row in the second matrix in (2.16) as follows.

$$
\left[\begin{array}{cc}
{\left[\begin{array}{cc}
b_{11}^{2} & b_{12} b_{13} \\
b_{21} b_{31} & b_{22} b_{33}
\end{array}\right]\left[\begin{array}{c}
A_{n ; 1}^{2} \\
A_{n ; 2} A_{n ; 3}
\end{array}\right]} & \left.\left[\begin{array}{ll}
b_{11} b_{12} & b_{12} b_{14} \\
b_{21} b_{32} & b_{22} b_{34}
\end{array}\right]\left[\begin{array}{c}
A_{n ; 1} A_{n ; 2} \\
A_{n ; 2} A_{n ; 4}
\end{array}\right]\right] \\
{\left[\begin{array}{ll}
b_{13} b_{11} & b_{14} b_{13} \\
b_{23} b_{31} & b_{24} b_{33}
\end{array}\right]\left[\begin{array}{c}
A_{n ; 3} A_{n ; 1} \\
A_{n ; 4} A_{n ; 3}
\end{array}\right]} & {\left[\begin{array}{ll}
b_{13} b_{12} & b_{14}^{2} \\
b_{23} b_{32} & b_{24} b_{34}
\end{array}\right]\left[\begin{array}{c}
A_{n ; 3} A_{n ; 2} \\
A_{n ; 4}^{2}
\end{array}\right]}
\end{array}\right]
$$

Applying (1.1), (1.2) and (2.1), (2.17) can be rewritten as

$$
\begin{gathered}
{\left[\begin{array}{cc}
{\left[\begin{array}{cc}
a_{11}^{2} & a_{12} a_{21} \\
a_{13} a_{31} & a_{14} a_{41}
\end{array}\right]\left[\begin{array}{c}
A_{n ; 11}^{2} \\
A_{n ; 12} A_{n ; 21}
\end{array}\right]} & \left.\left[\begin{array}{cc}
a_{11} a_{12} & a_{12} a_{22} \\
a_{13} a_{32} & a_{14} a_{42}
\end{array}\right]\left[\begin{array}{c}
A_{n ; 11} A_{n ; 12} \\
A_{n ; 12} A_{n ; 22}
\end{array}\right]\right] \\
{\left[\begin{array}{cc}
a_{21} a_{11} & a_{22} a_{21} \\
a_{23} a_{31} & a_{24} a_{41}
\end{array}\right]\left[\begin{array}{c}
A_{n ; 21} A_{n ; 11} \\
A_{n ; 22} A_{n ; 21}
\end{array}\right]} & {\left[\begin{array}{cc}
a_{21} a_{12} & a_{22}^{2} \\
a_{23} a_{32} & a_{24} a_{42}
\end{array}\right]\left[\begin{array}{c}
A_{n ; 21} A_{n ; 12} \\
A_{n ; 22}^{2}
\end{array}\right]}
\end{array}\right]} \\
=\left[\begin{array}{cc}
\left(B_{2 ; 11} \circ \tilde{A}_{2 ; 11}\right) X_{2, n ; 1} & \left(B_{2 ; 11} \circ \tilde{A}_{2 ; 12}\right) X_{2, n ; 2} \\
\left(B_{2 ; 12} \circ \tilde{A}_{2 ; 11}\right) X_{2, n ; 3} & \left(B_{2 ; 12} \circ \tilde{A}_{2 ; 12}\right) X_{2, n ; 4}
\end{array}\right] .
\end{gathered}
$$

Therefore, after the entries of $X_{2, n+1 ; 1}$ as in (2.17) or (2.18) have been permuted, $X_{2, n+1 ; 1}$ can be represented by a $2 \times 2$ matrix

$$
\hat{X}_{2, n+1 ; 1} \equiv \mathcal{P}\left(X_{2, n+1 ; 1}\right) \equiv\left[\begin{array}{ll}
X_{2, n+1 ; 1 ; 1} & X_{2, n+1 ; 1 ; 2} \\
X_{2, n+1 ; 1 ; 3} & X_{2, n+1 ; 1 ; 4}
\end{array}\right]
$$

where

$$
\left.\begin{array}{rl}
X_{2, n+1 ; 1 ; 1} & =S_{2 ; 11} X_{2, n ; 1}, \\
X_{2, n+1 ; 1 ; 2} & =S_{2 ; 12} X_{2, n ; 2}, \\
X_{2, n+1 ; 1 ; 3} & =S_{2 ; 13} X_{2, n ; 3}, \\
X_{2, n+1 ; 1 ; 4} & =S_{2 ; 14} X_{2, n ; 4}
\end{array}\right\}
$$

and

$$
\left.\begin{array}{l}
S_{2 ; 11}=B_{2 ; 11} \circ \tilde{A}_{2 ; 11} \equiv C_{2 ; 11}, \\
S_{2 ; 12}=B_{2 ; 11} \circ \tilde{A}_{2 ; 12} \equiv C_{2 ; 12}, \\
S_{2 ; 13}=B_{2 ; 12} \circ \tilde{A}_{2 ; 11} \equiv C_{2 ; 21}, \\
S_{2 ; 14}=B_{2 ; 12} \circ \tilde{A}_{2 ; 12} \equiv C_{2 ; 22},
\end{array}\right\} .
$$

The above derivation indicates that $X_{2, n+1 ; \alpha}$ can be reduced to $X_{2, n ; \beta}$ via multiplication with connecting matrices $C_{2 ; \alpha \beta}$. This procedure can be extended to introduce the connecting operator $\mathbb{C}_{m}=\left[C_{m ; \alpha \beta}\right]$, for all $m \geq 2$.

Before $\mathbb{C}_{m}$ is introduced, three products of matrices are defined as follows.

Definition 2.2. For any two matrices $\mathbb{M}=\left(M_{i j}\right)$ and $\mathbb{N}=\left(N_{k l}\right)$, the Kronecker product (tensor product) $\mathbb{M} \otimes \mathbb{N}$ of $\mathbb{M}$ and $\mathbb{N}$ is defined by

$$
\mathbb{M} \otimes \mathbb{N}=\left(M_{i j} \mathbb{N}\right)
$$

For any $n \geq 1$,

$$
\otimes \mathbb{N}^{n}=\mathbb{N} \otimes \mathbb{N} \otimes \cdots \otimes \mathbb{N}
$$


$n$-times in $\mathbb{N}$.

Next, for any two $m \times m$ matrices

$$
\mathbb{P}=\left(P_{i j}\right) \text { and } \mathbb{Q}=\left(Q_{i j}\right)
$$

where $P_{i j}$ and $Q_{i j}$ are numbers or matrices, the Hadamard product $\mathbb{P} \circ \mathbb{Q}$ is defined by

$$
\mathbb{P} \circ \mathbb{Q}=\left(P_{i j} \cdot Q_{i j}\right),
$$

where the product $P_{i j} \cdot Q_{i j}$ of $P_{i j}$ and $Q_{i j}$ may be a multiplication between numbers, between numbers and matrices or between matrices whenever it is well-defined.

Finally, product $\hat{\otimes}$ is defined as follows. For any $4 \times 4$ matrix

$$
\mathbb{M}_{2}=\left[\begin{array}{llll}
m_{11} & m_{12} & m_{21} & m_{22} \\
m_{13} & m_{14} & m_{23} & m_{24} \\
m_{31} & m_{32} & m_{41} & m_{42} \\
m_{33} & m_{34} & m_{43} & m_{44}
\end{array}\right]=\left[\begin{array}{ll}
M_{2 ; 1} & M_{2 ; 2} \\
M_{2 ; 3} & M_{2 ; 4}
\end{array}\right]
$$

and any $2 \times 2$ matrix

$$
\mathbb{N}=\left[\begin{array}{ll}
N_{1} & N_{2} \\
N_{3} & N_{4}
\end{array}\right]
$$

where $m_{i j}$ are numbers and $N_{k}$ are numbers or matrices, for $1 \leq i, j, k \leq 4$, define

$$
\mathbb{M}_{2} \hat{\otimes} \mathbb{N}=\left[\begin{array}{cccc}
m_{11} N_{1} & m_{12} N_{2} & m_{21} N_{1} & m_{22} N_{2} \\
m_{13} N_{3} & m_{14} N_{4} & m_{23} N_{3} & m_{24} N_{4} \\
m_{31} N_{1} & m_{32} N_{2} & m_{41} N_{1} & m_{42} N_{2} \\
m_{33} N_{3} & m_{34} N_{4} & m_{43} N_{3} & m_{44} N_{4}
\end{array}\right] .
$$

Furthermore, for $n \geq 1$, the $n+1$ th order of transition matrix of $\mathbb{M}_{2}$ is defined by

$$
\mathbb{M}_{n+1} \equiv \hat{\otimes} \mathbb{M}_{2}^{n}=\mathbb{M}_{2} \hat{\otimes} \mathbb{M}_{2} \hat{\otimes} \cdots \hat{\otimes} \mathbb{M}_{2}
$$

n-times in $\mathbb{M}_{2}$. More precisely,

$$
\begin{gathered}
\mathbb{M}_{n+1}=\mathbb{M}_{2} \hat{\otimes}\left(\hat{\otimes} \mathbb{M}_{2}^{n-1}\right)=\left[\begin{array}{lll}
M_{2 ; 1} \circ\left(\hat{\otimes} \mathbb{M}_{2}^{n-1}\right) & M_{2 ; 2} \circ\left(\hat{\otimes} \mathbb{M}_{2}^{n-1}\right) \\
M_{2 ; 3} \circ\left(\hat{\otimes} \mathbb{M}_{2}^{n-1}\right) & M_{2 ; 4} \circ\left(\hat{\otimes} \mathbb{M}_{2}^{n-1}\right)
\end{array}\right] \\
(2.27)=\left[\begin{array}{llll}
m_{11} M_{n ; 1} & m_{12} M_{n ; 2} & m_{21} M_{n ; 1} & m_{22} M_{n ; 2} \\
m_{13} M_{n ; 3} & m_{14} M_{n ; 4} & m_{23} M_{n ; 3} & m_{24} M_{n ; 4} \\
\hline m_{31} M_{n ; 1} & m_{32} M_{n ; 2} & m_{41} M_{n ; 1} & m_{42} M_{n ; 2} \\
m_{33} M_{n ; 3} & m_{34} M_{n ; 4} & m_{43} M_{n ; 3} & m_{44} M_{n ; 4}
\end{array}\right]=\left[\begin{array}{ll}
M_{n+1 ; 1} & M_{n+1 ; 2} \\
M_{n+1 ; 3} & M_{n+1 ; 4}
\end{array}\right],
\end{gathered}
$$

where

$$
\mathbb{M}_{n}=\hat{\otimes} \mathbb{M}_{2}^{n-1}=\left[\begin{array}{ll}
M_{n ; 1} & M_{n ; 2} \\
M_{n ; 3} & M_{n ; 4}
\end{array}\right]
$$

Here, the following convention is adopted,

$$
\hat{\otimes} \mathbb{M}_{2}^{0}=\mathbb{E}_{2 \times 2} .
$$


Definition 2.3. For $m \geq 2$, define

$$
\mathbb{C}_{m}=\left[\begin{array}{llll}
C_{m ; 11} & C_{m ; 12} & C_{m ; 13} & C_{m ; 14} \\
C_{m ; 21} & C_{m ; 22} & C_{m ; 23} & C_{m ; 24} \\
C_{m ; 31} & C_{m ; 32} & C_{m ; 33} & C_{m ; 34} \\
C_{m ; 41} & C_{m ; 42} & C_{m ; 43} & C_{m ; 44}
\end{array}\right]=\left[\begin{array}{cccc}
S_{m ; 11} & S_{m ; 12} & S_{m ; 21} & S_{m ; 22} \\
S_{m ; 13} & S_{m ; 14} & S_{m ; 23} & S_{m ; 24} \\
S_{m ; 31} & S_{m ; 32} & S_{m ; 41} & S_{m ; 42} \\
S_{m ; 33} & S_{m ; 34} & S_{m ; 43} & S_{m ; 44}
\end{array}\right],
$$

where

$$
\begin{aligned}
C_{m ; \alpha \beta} & =\left(\left[\begin{array}{ll}
a_{\alpha 1} & a_{\alpha 2} \\
a_{\alpha 3} & a_{\alpha 4}
\end{array}\right] \circ\left(\hat{\otimes}\left[\begin{array}{ll}
B_{2 ; 1} & B_{2 ; 2} \\
B_{2 ; 3} & B_{2 ; 4}
\end{array}\right]^{m-2}\right)_{2 \times 2}\right)_{2^{m-1} \times 2^{m-1}} \\
& \left(E_{2^{m-2} \times 2^{m-2}} \otimes\left(\left[\begin{array}{ll}
a_{1 \beta} & a_{2 \beta} \\
a_{3 \beta} & a_{4 \beta}
\end{array}\right]\right)\right)_{2^{m-1} \times 2^{m-1}} .
\end{aligned}
$$

Similarly, for $\mathbb{B}_{2}$, define

$$
\mathbb{U}_{m}=\left[\begin{array}{llll}
U_{m ; 11} & U_{m ; 12} & U_{m ; 13} & U_{m ; 14} \\
U_{m ; 21} & U_{m ; 22} & U_{m ; 23} & U_{m ; 24} \\
U_{m ; 31} & U_{m ; 32} & U_{m ; 33} & U_{m ; 34} \\
U_{m ; 41} & U_{m ; 42} & U_{m ; 43} & U_{m ; 44}
\end{array}\right]=\left[\begin{array}{llll}
W_{m ; 11} & W_{m ; 12} & W_{m ; 21} & W_{m ; 22} \\
W_{m ; 13} & W_{m ; 14} & W_{m ; 23} & W_{m ; 24} \\
W_{m ; 31} & W_{m ; 32} & W_{m ; 41} & W_{m ; 42} \\
W_{m ; 33} & W_{m ; 34} & W_{m ; 43} & W_{m ; 44}
\end{array}\right],
$$

where

$$
\begin{aligned}
U_{m ; \alpha \beta} & =\left(\left[\begin{array}{ll}
b_{\alpha_{1}} & b_{\alpha_{2}} \\
b_{\alpha_{3}} & b_{\alpha_{4}}
\end{array}\right] \circ\left(\hat{\otimes}\left[\begin{array}{ll}
A_{2 ; 1} & A_{2 ; 2} \\
A_{2 ; 3} & A_{2 ; 4}
\end{array}\right]^{m-2}\right)_{2 \times 2}\right)_{2^{m-1} \times 2^{m-1}} \\
& \circ\left(E_{2^{m-2} \times 2^{m-2}} \otimes\left(\left[\begin{array}{ll}
b_{1 \beta} & b_{2 \beta} \\
b_{3 \beta} & b_{4 \beta}
\end{array}\right]\right)\right)_{2^{m-1} \times 2^{m-1}} .
\end{aligned}
$$

$\mathbb{S}_{m}=\left[S_{m ; \alpha \beta}\right]$ and $\mathbb{W}_{m}=\left[W_{m ; \alpha \beta}\right]$.

Now $\mathbb{C}_{m+1}$ can be found from $\mathbb{C}_{m}$ by a recursive formula, as in (1.7).

Theorem 2.4. For any $m \geq 2$ and $1 \leq \alpha, \beta \leq 4$,

$$
C_{m+1 ; \alpha \beta}=\left[\begin{array}{cc}
a_{\alpha_{1}} C_{m ; 1 \beta} & a_{\alpha_{2}} C_{m ; 2 \beta} \\
a_{\alpha_{3}} C_{m ; 3 \beta} & a_{\alpha_{4}} C_{m ; 4 \beta}
\end{array}\right]
$$

and

$$
U_{m+1 ; \alpha \beta}=\left[\begin{array}{cc}
b_{\alpha_{1}} U_{m ; 1 \beta} & b_{\alpha_{2}} U_{m ; 2 \beta} \\
b_{\alpha_{3}} U_{m ; 3 \beta} & b_{\alpha_{4}} U_{m ; 4 \beta}
\end{array}\right] .
$$

Proof. By (2.27),

$$
\hat{\otimes} \mathbb{B}_{2}^{m-1}=\mathbb{B}_{2} \hat{\otimes}\left(\hat{\otimes} \mathbb{B}_{2}^{m-2}\right)=\left[\begin{array}{ll}
B_{2 ; 1} \circ\left(\hat{\otimes} \mathbb{B}_{2}^{m-2}\right) & B_{2 ; 2} \circ\left(\hat{\otimes} \mathbb{B}_{2}^{m-2}\right) \\
B_{2 ; 3} \circ\left(\hat{\otimes} \mathbb{B}_{2}^{m-2}\right) & B_{2 ; 4} \circ\left(\hat{\otimes} \mathbb{B}_{2}^{m-2}\right)
\end{array}\right] .
$$

Therefore,

$$
\begin{aligned}
& C_{m+1 ; \alpha \beta}=\left(B_{2 ; \alpha} \circ\left(\hat{\otimes} \mathbb{B}_{2}^{m-1}\right)\right) \circ\left(E_{2^{m-1} \times 2^{m-1}} \otimes \tilde{A}_{2 ; \beta}\right) \\
& =\left[\begin{array}{ll}
a_{\alpha 1}\left(B_{2 ; 1} \circ \hat{\otimes} \mathbb{B}_{2}^{m-2}\right) & a_{\alpha 2}\left(B_{2 ; 2} \circ \hat{\otimes} \mathbb{B}_{2}^{m-2}\right) \\
a_{\alpha 3}\left(B_{2 ; 3} \circ \hat{\otimes} \mathbb{B}_{2}^{m-2}\right) & a_{\alpha 4}\left(B_{2 ; 4} \circ \hat{\otimes} \mathbb{B}_{2}^{m-2}\right)
\end{array}\right] \circ\left(E_{2^{m-1} \times 2^{m-1}} \otimes \tilde{A}_{2 ; \beta}\right) \\
& =\left[\begin{array}{ll}
a_{\alpha 1} C_{m ; 1 \beta} & a_{\alpha 2} C_{m ; 2 \beta} \\
a_{\alpha 3} C_{m ; 3 \beta} & a_{\alpha 4} C_{m ; 4 \beta}
\end{array}\right] .
\end{aligned}
$$


A similar result also holds for $U_{m ; \alpha \beta}$; the details are omitted here. The proof is complete. $\mathrm{C}$

Notably, (2.32) implies $\mathbb{C}_{m ; i j}$ is $a_{i_{1} i_{2}} a_{i_{2} i_{3}} \cdots a_{i_{m} i_{m+1}}$ with $i_{1}=i$ and $i_{m+1}=j$. $\mathbb{C}_{m ; i j}$ consist of all words(or paths) of length $m$ starting from $i$ and ending at $j$. Indeed, the entries of $\mathbb{C}_{m}$ and $\mathbb{B}_{m+1}$ are the same. However, the arrangements are different. $\mathbb{C}_{m}$ can also be used to study the primitivity of $\mathbb{A}_{n}, n \geq 2$, as in [6].

That the recursive formula (1.24) holds remains to be shown. Indeed, in (2.6) substituting $n$ for $n+1$ and using (1.7),

$$
\begin{aligned}
& A_{m, n+1 ; \alpha}^{(k)} \\
& =A_{n+1 ; j_{1} j_{2}} A_{n+1 ; j_{2} j_{3}} \cdots A_{n+1, j_{m} j_{m+1}} \\
& =\prod_{i=1}^{m}\left[\begin{array}{ll}
b_{\alpha_{i} 1} A_{n ; 11} & b_{\alpha_{i} 2} A_{n ; 12} \\
b_{\alpha_{i} 3} A_{n ; 21} & b_{\alpha_{i} 4} A_{n ; 22}
\end{array}\right]
\end{aligned}
$$

where $\alpha_{i}=\alpha\left(j_{i}, j_{i+1}\right)$, for $1 \leq i \leq m$. After $m$ matrix multiplications are executed in $(2.34)$,

$$
A_{m, n+1 ; \alpha}^{(k)}=\left[\begin{array}{cc}
A_{m, n+1 ; \alpha ; 1}^{(k)} & A_{m, n+1 ; \alpha ; 2}^{(k)} \\
A_{m, n+1 ; \alpha ; 3}^{(k)} & A_{m, n+1 ; \alpha ; 4}^{(k)}
\end{array}\right]
$$

where

$$
A_{m, n+1 ; \alpha ; \beta}^{(k)}=\sum_{l=1}^{2^{m-1}} K(m ; \alpha, \beta ; k, l) A_{m, n ; \beta}^{(l)}
$$

is a linear combination of $A_{m, n ; \beta}^{(l)}$ with the coefficients $K(m ; \alpha, \beta ; k, l)$ which are products of $b_{\alpha_{l} j}, 1 \leq l \leq m$. $K(m ; \alpha, \beta ; k, l)$ must be studied in more details.

Note that

$$
\begin{gathered}
\mathbb{A}_{n+1}^{m}=\left[\begin{array}{ll}
A_{m, n+1 ; 1} & A_{m, n+1 ; 2} \\
A_{m, n+1 ; 3} & A_{m, n+1 ; 4}
\end{array}\right] \\
=\left[\begin{array}{ll}
\sum_{k=1}^{2^{m-1}} A_{m, n+1 ; 1}^{(k)} & \sum_{k=1}^{2^{m-1}} A_{m, n+1 ; 2}^{(k)} \\
\sum_{k=1}^{2^{m-1}} A_{m, n+1 ; 3}^{(k)} & \sum_{k=1}^{2^{m-1}} A_{m, n+1 ; 4}^{(k)}
\end{array}\right]
\end{gathered}
$$$$
=\left[\begin{array}{llllll}
\sum_{k=1}^{2^{m-1}} A_{m, n+1 ; 1 ; 1}^{(k)} & \sum_{k=1}^{2^{m-1}} A_{m, n+1 ; 1 ; 2}^{(k)} & \sum_{k=1}^{2^{m-1}} A_{m, n+1 ; 2 ; 1}^{(k)} & \sum_{k=1}^{2^{m-1}} A_{m, n+1 ; 2 ; 2}^{(k)} \\
\sum_{k=1}^{2^{m-1}} A_{m, n+1 ; 1 ; 3}^{(k)} & \sum_{k=1}^{2^{m-1}} A_{m, n+1 ; 1 ; 4}^{(k)} & \sum_{k=1}^{2^{m-1}} A_{m, n+1 ; 2 ; 3}^{(k)} & \sum_{k=1}^{2^{m}-1} A_{m, n+1 ; 2 ; 4}^{(k)} \\
\sum_{k=1}^{2^{m-1}} A_{m, n+1 ; 3 ; 1}^{(k)} & \sum_{k=1}^{2^{m-1}} A_{m, n+1 ; 3 ; 2}^{(k)} & \sum_{k=1}^{2^{m-1}} A_{m, n+1 ; 4 ; 1}^{(k)} & \sum_{k=1}^{2^{m}-1} A_{m, n+1 ; 4 ; 2}^{(k)} \\
\sum_{k=1}^{2^{m-1}} A_{m, n+1 ; 3 ; 3}^{(k)} & \sum_{k=1}^{2^{m-1}} A_{m, n+1 ; 3 ; 4}^{(k)} & \sum_{k=1}^{2^{m-1}} A_{m, n+1 ; 4 ; 3}^{(k)} & \sum_{k=1}^{2^{m}-1} A_{m, n+1 ; 4 ; 4}^{(k)}
\end{array}\right]
$$

Now, $X_{m, n+1 ; \alpha ; \beta}$ is defined as

$$
X_{m, n+1 ; \alpha ; \beta}=\left(A_{m, n+1 ; \alpha ; \beta}^{(k)}\right)^{t} .
$$


As in (2.17), the entries of $X_{m, n+1 ; \alpha}$ are rearranged into a new matrix

$$
\hat{X}_{m, n+1 ; \alpha} \equiv \mathcal{P}\left(X_{m, n+1 ; \alpha}\right) \equiv\left[\begin{array}{cc}
X_{m, n+1 ; \alpha ; 1} & X_{m, n+1 ; \alpha ; 2} \\
X_{m, n+1 ; \alpha ; 3} & X_{m, n+1 ; \alpha ; 4}
\end{array}\right] .
$$

From (2.36) and (2.38),

$$
X_{m, n+1 ; \alpha ; \beta}=\mathbb{K}(m ; \alpha, \beta) X_{m, n ; \beta}
$$

where

$$
\mathbb{K}(m ; \alpha, \beta)=(K(m ; \alpha, \beta ; k, l)), 1 \leq k, l \leq 2^{m-1},
$$

is a $2^{m-1} \times 2^{m-1}$ matrix. Now, $\mathbb{K}(m ; \alpha, \beta)=S_{m ; \alpha \beta}$ must be shown as follows.

TheOrem 2.5. For any $m \geq 2$ and $n \geq 2$, let $S_{m ; \alpha \beta}$ be given as in (2.28) and (2.29). Then,

$$
\mathbb{K}(m ; \alpha, \beta)=S_{m ; \alpha \beta},
$$

i.e.,

$$
X_{m, n+1 ; \alpha ; \beta}=S_{m ; \alpha \beta} X_{m, n ; \beta},
$$

or equivalently, the recursive formula (1.24) holds. That is,

$$
A_{m, n+1 ; \alpha}^{(k)}=\left[\begin{array}{ll}
\sum_{l=1}^{2^{m-1}}\left(S_{m ; \alpha 1}\right)_{k l} A_{m, n ; 1}^{(l)} & \sum_{l=1}^{2^{m-1}}\left(S_{m ; \alpha 2}\right)_{k l} A_{m, n ; 2}^{(l)} \\
\sum_{l=1}^{2^{m-1}}\left(S_{m ; \alpha 3}\right)_{k l} A_{m, n ; 3}^{(l)} & \sum_{l=1}^{2^{m-1}}\left(S_{m ; \alpha 4}\right)_{k l} A_{m, n ; 4}^{(l)}
\end{array}\right] .
$$

Moreover, for $n=1$,

$$
A_{m, 2 ; \alpha}^{(k)}=\left[\begin{array}{ll}
\sum_{l=1}^{2^{m-1}}\left(S_{m ; \alpha 1}\right)_{k l} & \sum_{l=1}^{2^{m-1}}\left(S_{m ; \alpha 2}\right)_{k l} \\
\sum_{l=1}^{2^{m-1}}\left(S_{m ; \alpha 3}\right)_{k l} & \sum_{l=1}^{2^{m-1}}\left(S_{m ; \alpha 4}\right)_{k l}
\end{array}\right]
$$

for any $1 \leq k \leq 2^{m-1}$ and $\alpha \in\{1,2,3,4\}$.

Proof. The result is proven by the induction on $m$.

When $m=2$, and $\alpha=1,(2.41)$ was proven as in Example 2.1. The case with $\alpha=2,3$ and 4 can also be proved analogously; the details are omitted.

Now, (2.41) ia assumed to hold for $m$; the goal is to show that it also holds for $m+1$. Since

$$
\mathbb{A}_{n+1}^{m+1}=\mathbb{A}_{n+1} \cdot \mathbb{A}_{n+1}^{m}=\left[\begin{array}{ll}
A_{n+1 ; 1} & A_{n+1 ; 2} \\
A_{n+1 ; 3} & A_{n+1 ; 4}
\end{array}\right]\left[\begin{array}{ll}
A_{m, n+1,1} & A_{m, n+1 ; 2} \\
A_{m, n+1,3} & A_{m, n+1 ; 4}
\end{array}\right],
$$


(2.11) implies

$$
\begin{aligned}
& X_{m+1, n+1 ; 1}=\left[\begin{array}{l}
A_{n+1 ; 1} X_{m, n+1 ; 1} \\
A_{n+1 ; 2} X_{m, n+1 ; 3}
\end{array}\right], X_{m+1, n+1 ; 2}=\left[\begin{array}{c}
A_{n+1 ; 1} X_{m, n+1 ; 2} \\
A_{n+1 ; 2} X_{m, n+1 ; 4}
\end{array}\right], \\
& X_{m+1, n+1 ; 3}=\left[\begin{array}{c}
A_{n+1 ; 3} X_{m, n+1 ; 1} \\
A_{n+1 ; 4} X_{m, n+1 ; 3}
\end{array}\right], \text { and } X_{m+1, n+1 ; 4}=\left[\begin{array}{c}
A_{n+1 ; 3} X_{m, n+1 ; 2} \\
A_{n+1 ; 4} X_{m, n+1 ; 4}
\end{array}\right] .
\end{aligned}
$$

For $\alpha=1$, by induction on $m$,

$$
\begin{aligned}
& \left(A_{n+1 ; 1} \mathcal{P}\left(X_{m, n+1 ; 1}\right), A_{n+1 ; 2} \mathcal{P}\left(X_{m, n+1 ; 3}\right)\right)^{t} \\
& =\left[\begin{array}{ll}
{\left[\begin{array}{ll}
b_{11} A_{n ; 1} & b_{12} A_{n ; 2} \\
b_{13} A_{n ; 3} & b_{14} A_{n ; 4}
\end{array}\right]} & {\left[\begin{array}{ll}
S_{m ; 11} X_{m, n ; 1} & S_{m ; 12} X_{m, n ; 2} \\
S_{m ; 13} X_{m, n ; 3} & S_{m ; 14} X_{m, n ; 4}
\end{array}\right]} \\
{\left[\begin{array}{ll}
b_{21} A_{n ; 1} & b_{22} A_{n ; 2} \\
b_{23} A_{n ; 3} & b_{24} A_{n ; 4}
\end{array}\right]} & \left.\begin{array}{ll}
S_{m ; 31} X_{m, n ; 1} & S_{m ; 32} X_{m, n ; 2} \\
S_{m ; 33} X_{m, n ; 3} & S_{m ; 34} X_{m, n ; 4}
\end{array}\right]
\end{array}\right] \\
& =\left[\begin{array}{ll}
{\left[\begin{array}{ll}
b_{11} S_{m ; 11} A_{n ; 1} X_{m, n ; 1} & b_{11} S_{m ; 12} A_{n ; 1} X_{m, n ; 2} \\
b_{13} S_{m ; 11} A_{n ; 3} X_{m, n ; 1} & b_{13} S_{m ; 12} A_{n ; 3} X_{m, n ; 2}
\end{array}\right]} \\
{\left[\begin{array}{ll}
b_{21} S_{m ; 31} A_{n ; 1} X_{m, n ; 1} & b_{21} S_{m ; 32} A_{n ; 1} X_{m, n ; 2} \\
b_{23} S_{m ; 31} A_{n ; 3} X_{m, n ; 1} & b_{23} S_{m ; 32} A_{n ; 3} X_{m, n ; 2}
\end{array}\right]}
\end{array}\right] \\
& +\left[\begin{array}{ll}
{\left[\begin{array}{ll}
b_{12} S_{m ; 13} A_{n ; 2} X_{m, n ; 3} & b_{12} S_{m ; 14} A_{n ; 2} X_{m, n ; 4} \\
b_{14} S_{m ; 13} A_{n ; 4} X_{m, n ; 3} & b_{14} S_{m ; 14} A_{n ; 4} X_{m, n ; 4}
\end{array}\right]} \\
{\left[\begin{array}{ll}
b_{22} S_{m ; 33} A_{n ; 2} X_{m, n ; 3} & b_{22} S_{m ; 34} A_{n ; 2} X_{m, n ; 4} \\
b_{24} S_{m ; 33} A_{n ; 4} X_{m, n ; 3} & b_{24} S_{m ; 34} A_{n ; 4} X_{m, n ; 4}
\end{array}\right]}
\end{array}\right]
\end{aligned}
$$

Hence $X_{m+1, n+1 ; 1}$ can be represented by a matrix

$$
\begin{aligned}
& \hat{X}_{m+1, n+1 ; 1} \equiv \mathcal{P}\left(X_{m+1, n+1 ; 1}\right) \equiv\left[\begin{array}{ll}
X_{m+1, n+1 ; 1,1} & X_{m+1, n+1 ; 1,2} \\
X_{m+1, n+1 ; 1,3} & X_{m+1, n+1 ; 1,4}
\end{array}\right] \\
& =\left[\begin{array}{cc}
{\left[\begin{array}{cc}
b_{11} S_{m ; 11} & b_{12} S_{m ; 13} \\
b_{21} S_{m ; 31} & b_{22} S_{m ; 33}
\end{array}\right]\left[\begin{array}{l}
A_{n ; 1} X_{m, n ; 1} \\
A_{n ; 2} X_{m, n ; 3}
\end{array}\right]} & {\left[\begin{array}{ll}
b_{11} S_{m ; 12} & b_{12} S_{m ; 14} \\
b_{21} S_{m ; 32} & b_{22} S_{m ; 34}
\end{array}\right]\left[\begin{array}{l}
A_{n ; 1} X_{m, n ; 2} \\
A_{n ; 2} X_{m, n ; 4}
\end{array}\right]} \\
{\left[\begin{array}{ll}
b_{13} S_{m ; 11} & b_{14} S_{m ; 13} \\
b_{23} S_{m ; 31} & b_{24} S_{m ; 33}
\end{array}\right]\left[\begin{array}{l}
A_{n ; 3} X_{m, n ; 1} \\
A_{n ; 4} X_{m, n ; 3}
\end{array}\right]} & {\left[\begin{array}{ll}
b_{13} S_{m ; 12} & b_{14} S_{m ; 14} \\
b_{23} S_{m ; 32} & b_{24} S_{m ; 34}
\end{array}\right]\left[\begin{array}{l}
A_{n ; 3} X_{m, n ; 2} \\
A_{n ; 4} X_{m, n ; 4}
\end{array}\right]}
\end{array}\right]
\end{aligned}
$$

Once again, (1.1), (1.2) and (2.1) can be used to recast the matrix $\hat{X}_{m+1, n+1 ; 1}$ as

$$
\left[\begin{array}{c}
{\left[\begin{array}{ll}
a_{11} C_{m ; 11} & a_{12} C_{m ; 21} \\
a_{13} C_{m ; 31} & a_{14} C_{m ; 41}
\end{array}\right] X_{m+1, n ; 1}\left[\begin{array}{ll}
a_{11} C_{m ; 12} & a_{12} C_{m ; 22} \\
a_{13} C_{m ; 32} & a_{14} C_{m ; 42}
\end{array}\right] X_{m+1, n ; 2}} \\
{\left[\begin{array}{ll}
a_{21} C_{m ; 11} & a_{22} C_{m ; 21} \\
a_{23} C_{m ; 31} & a_{24} C_{m ; 41}
\end{array}\right] X_{m+1, n ; 3}\left[\begin{array}{ll}
a_{21} C_{m ; 12} & a_{22} C_{m ; 22} \\
a_{23} C_{m ; 32} & a_{24} C_{m ; 42}
\end{array}\right] X_{m+1, n ; 4}}
\end{array}\right]
$$

According to Theorem 2.4, the above matrix becomes

$$
=\left[\begin{array}{ll}
C_{m+1 ; 11} X_{m+1, n ; 1} & C_{m+1 ; 12} X_{m+1, n ; 2} \\
C_{m+1 ; 21} X_{m+1, n ; 3} & C_{m+1 ; 22} X_{m+1, n ; 4}
\end{array}\right]=\left[\begin{array}{ll}
S_{m+1 ; 11} X_{m+1, n ; 1} & S_{m+1 ; 12} X_{m+1, n ; 2} \\
S_{m+1 ; 13} X_{m+1, n ; 3} & S_{m+1 ; 14} X_{m+1, n ; 4}
\end{array}\right] .
$$


The cases with $\alpha=2,3$ and 4 can also be considered analogously (2.41) follows. Next, (2.42) follows easily from (2.35), (2.36) and (2.41).

Equation (2.43) remains to be shown. If the $2 \times 2$ matrix

$$
\mathbb{A}_{1} \equiv\left[\begin{array}{cc}
A_{1 ; 11} & A_{1 ; 12} \\
A_{1 ; 21} & A_{1 ; 22}
\end{array}\right] \equiv\left[\begin{array}{cc}
A_{1 ; 1} & A_{1 ; 2} \\
A_{1 ; 3} & A_{1 ; 4}
\end{array}\right] \equiv\left[\begin{array}{cc}
1 & 1 \\
1 & 1
\end{array}\right]
$$

is introduced, then the previous argument also hold for $n=1$. Hence, (2.43) holds. The proof is complete.

For any positive integer $p \geq 2$, applying Theorem $2.5 p$ times permits the elementary patterns of $\mathbb{A}_{n+p}^{m}$ to be expressed as the product of a sequence of $S_{m ; \beta_{i} \beta_{i+1}}$ and the elementary patterns in $\mathbb{A}_{n}^{m}$. The elementary pattern in $\mathbb{A}_{n+p}^{m}$ is first studied.

For any $p \geq 2$ and $1 \leq q \leq p-1$, define

$$
A_{m, n+p ; \alpha ; \beta_{1} ; \beta_{2} ; \cdots ; \beta_{q}}^{(k)}=\left[\begin{array}{ll}
A_{m, n+p ; \alpha ; \beta_{1} ; \beta_{2} ; \cdots ; \beta_{q} ; 1}^{(k)} & A_{m, n+p ; \alpha ; \beta_{1} ; \beta_{2} ; \cdots ; \beta_{q} ; 2}^{(k)} \\
A_{m, n+p ; \alpha ; \beta_{1} ; \beta_{2} ; \cdots ; \beta_{q} ; 3}^{(k)} & A_{m, n+p ; \alpha ; \beta_{1} ; \beta_{2} ; \cdots ; \beta_{q} ; 4}^{(k)}
\end{array}\right] .
$$

Then

$$
A_{m, n+p ; \alpha ; \beta_{1} ; \beta_{2} ; \cdots ; \beta_{p}}^{(k)}=\sum_{l_{1}=1}^{2^{m-1}} \cdots \sum_{l_{p}=1}^{2^{m-1}}\left(\prod_{i=1}^{p} K\left(m ; \beta_{i-1}, \beta_{i} ; l_{i-1}, l_{i}\right)\right) A_{m, n ; \beta_{p}}^{\left(l_{p}\right)}
$$

where $\beta_{0}=\alpha$ and $l_{0}=k$ can be easily verified. Therefore, for any $p \geq 1$, a generalization for $(2.37)$ can be found for $\mathbb{A}_{n+p}^{m}$ as a $2^{p+1} \times 2^{p+1}$ matrix

$$
\mathbb{A}_{n+p}^{m}=\left[A_{m, n+p ; \alpha ; \beta_{1} ; \beta_{2} \cdots ; \beta_{p}}\right]
$$

where

$$
A_{m, n+p ; \alpha ; \beta_{1} ; \beta_{2} \cdots ; \beta_{p}}=\sum_{k=1}^{2^{m-1}} A_{m, n ; \alpha ; \beta_{1} ; \beta_{2} \cdots ; \beta_{p}}^{(k)}
$$

In particular, if $\alpha ; \beta_{1}, \beta_{2} \cdots, \beta_{p} \in\{1,4\}$, then $A_{m, n+p ; \alpha ; \beta_{1} ; \beta_{2} \cdots ; \beta_{p}}$ lies on the diagonal of $\mathbb{A}_{n+p}^{m}$ in (2.47).

Now, define

$$
X_{m, n+p ; \alpha ; \beta_{1} ; \beta_{2} ; \cdots ; \beta_{p}}=\left(A_{m, n+p ; \alpha ; \beta_{1} ; \beta_{2} ; \cdots ; \beta_{p}}^{(k)}\right)^{t}
$$

Therefore, Theorem 2.5 can be generalized to

TheOREM 2.6. For any $m \geq 2, n \geq 2$ and $p \geq 1$,

$$
X_{m, n+p ; \alpha ; \beta_{1} ; \beta_{2} \cdots ; \beta_{p}}=S_{m ; \alpha \beta_{1}} S_{m ; \beta_{1} \beta_{2}} \cdots S_{m ; \beta_{p-1} \beta_{p}} X_{m, n ; \beta_{p}}
$$

where $\alpha, \beta_{i} \in\{1,2,3,4\}$ and $1 \leq i \leq p$. 
Proof. From (2.46), (2.40) and (2.42),

$$
\begin{aligned}
& A_{m, n+p ; \alpha ; \beta_{1} ; \beta_{2} ; \cdots ; \beta_{p}}^{(k)}=\sum_{l_{1}=1}^{2^{m-1}} \cdots \sum_{l_{p}=1}^{2^{m-1}}\left(\prod_{i=1}^{p} K\left(m ; \beta_{i-1}, \beta_{i} ; l_{i-1}, l_{i}\right)\right) A_{m, n ; \beta_{p}}^{\left(l_{p}\right)} \\
& =\sum_{l_{1}=1}^{2^{m-1}} \cdots \sum_{l_{p}=1}^{2^{m-1}}\left(\prod_{i=1}^{p}\left(S_{m ; \beta_{i-1} \beta_{i}}\right)_{l_{i-1} l_{i}}\right) A_{m, n ; \beta_{p}}^{\left(l_{p}\right)} \\
& =\sum_{l_{1}=1}^{2^{m-1}} \cdots \sum_{l_{p}=1}^{2^{m-1}}\left(S_{m ; \beta_{0} \beta_{1}}\right)_{l_{0} l_{1}}\left(S_{m ; \beta_{1} \beta_{2}}\right)_{l_{1} l_{2}} \cdots\left(S_{m ; \beta_{p-1} \beta_{p}}\right)_{l_{p-1} l_{p}} A_{m, n ; \beta_{p}}^{\left(l_{p}\right)} \\
& =\sum_{l_{p}=1}^{2^{m-1}}\left(S_{m ; \beta_{0} \beta_{1}} S_{m ; \beta_{1} \beta_{2}} \cdots S_{m ; \beta_{p-1} \beta_{p}}\right)_{l_{0} l_{p}} A_{m, n ; \beta_{p}}^{\left(l_{p}\right)} \\
& =\sum_{l_{p}=1}\left(S_{m ; \alpha \beta_{1}} S_{m ; \beta_{1} \beta_{2}} \cdots S_{m ; \beta_{p-1} \beta_{p}}\right)_{k l_{p}} A_{m, n ; \beta_{p}}^{\left(l_{p}\right)}
\end{aligned}
$$

is derived. By (2.49), then

$$
\begin{aligned}
& X_{m, n+p ; \alpha ; \beta_{1} ; \beta_{2} ; \cdots ; \beta_{p}}=\left(A_{m, n+p ; \alpha ; \beta_{1} ; \beta_{2} ; \cdots ; \beta_{p}}^{(k)}\right)^{t} \\
& =\left(\sum_{l_{p}=1}^{2^{m-1}}\left(S_{m ; \alpha \beta_{1}} S_{m ; \beta_{1} \beta_{2}} \cdots S_{m ; \beta_{p-1} \beta_{p}}\right)_{k l_{p}} A_{m, n ; \beta_{p}}^{\left(l_{p}\right)}\right)^{t} \\
& =S_{m ; \alpha \beta_{1}} S_{m ; \beta_{1} \beta_{2}} \cdots S_{m ; \beta_{p-1} \beta_{p}} X_{m, n ; \beta_{p}} .
\end{aligned}
$$

The proof is complete.

2.2. Lower bound of entropy. In this subsection, the connecting operator $\mathbb{C}_{m}$ is employed to estimate the lower bound of entropy, and in particular, to verify the positivity of entropy.

First, recall some properties of $\Gamma_{m, n}$ and spatial entropy.

$\Gamma_{m, n}$ satisfies the subadditivity in $m$ and $n$ :

$$
\Gamma_{m_{1}+m_{2}, n} \leq \Gamma_{m_{1}, n} \Gamma_{m_{2}, n},
$$

and

$$
\Gamma_{m, n_{1}+n_{2}} \leq \Gamma_{m, n_{1}} \Gamma_{m, n_{2}}
$$

or equivalently,

$$
\left|\mathbb{A}_{n}^{m_{1}+m_{2}}\right| \leq\left|\mathbb{A}_{n}^{m_{1}}\right|\left|\mathbb{A}_{n}^{m_{2}}\right|
$$

and

$$
\left|\mathbb{A}_{n_{1}+n_{2}}^{m}\right| \leq\left|\mathbb{A}_{n_{1}}^{m}\right|\left|\mathbb{A}_{n_{2}}^{m}\right|,
$$

for positive integers $m, n, m_{1}, n_{1}, m_{2}$ and $n_{2}$. Here

$$
\mathbb{A}_{1}=\left[\begin{array}{ll}
1 & 1 \\
1 & 1
\end{array}\right]
$$

is applied. 
The subadditivity property implies

$$
\limsup _{m, n \rightarrow \infty} \frac{1}{m n} \log \left|\mathbb{A}_{n}^{m}\right| \leq \frac{1}{p q} \log \left|\mathbb{A}_{q}^{p-1}\right|
$$

for any $p$ and $q \geq 2$. Therefore,

$$
h\left(\mathbb{A}_{2}\right)=\lim _{m, n \rightarrow \infty} \frac{1}{m n} \log \left|\mathbb{A}_{n}^{m}\right|
$$

exists, and equals

$$
\inf _{p, q \geq 2} \frac{1}{p q} \log \left|\mathbb{A}_{q}^{p-1}\right|
$$

In particular, $h\left(\mathbb{A}_{2}\right)$ has an upper bound

$$
h\left(\mathbb{A}_{2}\right) \leq \frac{1}{p q} \log \left|\mathbb{A}_{q}^{p-1}\right|
$$

for any $p$ and $q \geq 2$.

Similarly, when $\mathbb{A}_{2}$ is horizontal (or vertical) transition matrix for any $m \geq 1$ and $q \geq 2$,

$$
\limsup _{n \rightarrow \infty} \frac{1}{n} \log \left|\mathbb{A}_{n}^{m}\right| \leq \frac{1}{q} \log \left|\mathbb{A}_{q}^{m}\right| .
$$

Hence, the spatial entropy is $h_{m}\left(\mathbb{A}_{2}\right)$ on an infinite lattice $\mathbb{Z}_{m+1 \times \infty}\left(\right.$ or $\left.\mathbb{Z}_{\infty \times m+1}\right)$ and

$$
h_{m}\left(\mathbb{A}_{2}\right) \equiv \lim _{n \rightarrow \infty} \frac{1}{n} \log \left|\mathbb{A}_{n}^{m}\right|=\inf _{q \geq 2} \frac{1}{q} \log \left|\mathbb{A}_{q}^{m}\right| .
$$

For the proof of the above results, see [15].

Furthermore, by Perron-Frobenius theorem,

$$
\lim _{m \rightarrow \infty} \frac{1}{m} \log \left|\mathbb{A}_{n}^{m}\right|=\log \rho\left(\mathbb{A}_{n}\right) .
$$

Therefore, for any $n \geq 2$

$$
h\left(\mathbb{A}_{2}\right) \leq \frac{1}{n} \log \rho\left(\mathbb{A}_{n}\right) .
$$

For a proof of (2.61), see [4], [30].

The following notation is adopted.

Definition 2.7. Let $X=\left(X_{1}, \cdots, X_{M}\right)^{t}$, where $X_{k}$ are $N \times N$ matrices. Define the summation of $X_{k}$ by

$$
|X|=\sum_{k=1}^{N} X_{k}
$$

If $\mathbb{M}=\left[M_{i j}\right]$ is a $M \times M$ matrix, then

$$
|\mathbb{M} X|=\sum_{i=1}^{M} \sum_{j=1}^{M} M_{i j} X_{j}
$$


Note that, (2.63) implies

$$
\left|X_{m, n ; \alpha}\right|=\sum_{k=1}^{2^{m-1}} A_{m, n ; \alpha}^{(k)}=A_{m, n ; \alpha} .
$$

As usual, the set of all matrices with the same order can be partially ordered.

Definition 2.8. Let $\mathbb{M}=\left[M_{i j}\right]$ and $\mathbb{N}=\left[N_{i j}\right]$ be two $M \times M$ matrices, $\mathbb{M} \geq \mathbb{N}$ if $M_{i j} \geq N_{i j}$ for all $1 \leq i, j \leq M$.

Notably, if $\mathbb{A}_{2} \geq \mathbb{A}_{2}^{\prime}$ then $\mathbb{A}_{n} \geq \mathbb{A}_{n}^{\prime}$ for all $n \geq 2$. Therefore, $h\left(\mathbb{A}_{2}\right) \geq h\left(\mathbb{A}_{2}^{\prime}\right)$. Hence, the spatial entropy as a function of $\mathbb{A}_{2}$ is monotonic with respect to the partial order $\geq$.

Definition 2.9. A K 1 multiple index

$$
\mathcal{B}_{K} \equiv\left(\beta_{1} \beta_{2} \cdots \beta_{K} \beta_{K+1}\right)
$$

is called a (periodic) cycle if

$$
\beta_{K+1}=\beta_{1} .
$$

It is called a diagonal cycle if (2.67) holds and

$$
\beta_{k} \in\{1,4\}
$$

for each $1 \leq k \leq K+1$.

For a diagonal cycle (2.66), denote

$$
\bar{\beta}_{K}=\beta_{1} ; \beta_{2} ; \cdots ; \beta_{K}
$$

and

$$
\bar{\beta}_{K}^{n}=\bar{\beta}_{K} ; \bar{\beta}_{K} ; \cdots ; \bar{\beta}_{K} \cdot(\text { n times })
$$

First, prove the following Lemma.

Lemma 2.10. Let $m \geq 2, K \geq 1, \mathcal{B}_{K}$ be a diagonal cycle. Then, for any $n \geq 1$,

$$
\rho\left(\mathbb{A}_{n K+2}^{m}\right) \geq \rho\left(\left|\left(S_{m ; \beta_{1} \beta_{2}} S_{m ; \beta_{2} \beta_{3}} \cdots S_{m ; \beta_{K} \beta_{K+1}}\right)^{n} X_{m, 2 ; \beta_{1}}\right|\right) .
$$

Proof. Since $\mathcal{B}_{K}$ is a periodic cycle, Theorem 2.6 implies

$$
X_{m, n K+2 ; \bar{\beta}_{K}^{n}}=\left(S_{m ; \beta_{1} \beta_{2}} S_{m ; \beta_{2} \beta_{3}} \cdots S_{m ; \beta_{K} \beta_{K+1}}\right)^{n} X_{m, 2 ; \beta_{1}} .
$$

Furthermore $\mathcal{B}_{K}$ is diagonal, and $\left|X_{m, n K+2 ; \bar{\beta}_{k}^{n}}\right|=A_{m, n K+2 ; \bar{\beta}_{k}^{n}}$ lies on the diagonal part as in (2.47) with $n+p=n K+2$, therefore

$$
\rho\left(\mathbb{A}_{n K+2}^{m}\right) \geq \rho\left(\left|X_{m, n K+2 ; \bar{\beta}_{K}^{n}}\right|\right) .
$$

Therefore, (2.71) follows from (2.72) and (2.73).

The proof is complete.

The following lemma is valuable in studying maximum eigenvalue of $\left(S_{m ; \beta_{1} \beta_{2}} \cdots S_{m ; \beta_{K} \beta_{K+1}}\right)^{n} X_{m, 2 ; \beta_{1}}$ in $(2.71)$. 
Lemma 2.11. For any $m \geq 2,1 \leq k \leq 2^{m-1}$ and $\alpha \in\{1,4\}$, if

$$
\operatorname{tr}\left(A_{m, 2 ; \alpha}^{(k)}\right)=0
$$

then for all $1 \leq l \leq 2^{m-1}$,

$$
\left(S_{m, \alpha 1}\right)_{k l}=0 \text { and }\left(S_{m ; \alpha 4}\right)_{k l}=0,
$$

i.e., the $k$-th rows of matrices $S_{m ; \alpha 1}$ and $S_{m ; \alpha 4}$ are zeros. Furthermore, for any diagonal cycle $\mathcal{B}_{K}$, let $U=\left(u_{1}, u_{2}, \cdots, u_{2^{m-1}}\right)$ be an eigenvector of $S_{m ; \beta_{1} \beta_{2}} S_{m ; \beta_{2} \beta_{3}} \cdots S_{m ; \beta_{K} \beta_{1}}$, if $u_{k} \neq 0$ for some $1 \leq k \leq 2^{m-1}$, then

$$
\operatorname{tr}\left(A_{m, 2 ; \alpha}^{(k)}\right)>0 .
$$

Proof. Since $A_{m, 2 ; \alpha}^{(k)}$ can be expressed as in (2.43). Therefore, $\operatorname{tr}\left(A_{m, 2 ; \alpha}^{(k)}\right)=0$ if and only if (2.75) holds for all $1 \leq l \leq 2^{m-1}$. The second part of the lemma follows easily from the first part.

The proof is complete.

By Lemma 2.10 and Lemma 2.11, the lower bound of entropy can be obtained as follows.

Theorem 2.12. Let $\beta_{1} \beta_{2} \cdots \beta_{K} \beta_{1}$ be a diagonal cycle. Then for any $m \geq 2$,

$$
h\left(\mathbb{A}_{2}\right) \geq \frac{1}{m K} \log \rho\left(S_{m ; \beta_{1} \beta_{2}} S_{m ; \beta_{2} \beta_{3}} \cdots S_{m ; \beta_{K} \beta_{1}}\right) .
$$

and

$$
h\left(\mathbb{A}_{2}\right) \geq \frac{1}{m K} \log \rho\left(W_{m ; \beta_{1} \beta_{2}} W_{m ; \beta_{2} \beta_{3}} \cdots W_{m ; \beta_{K} \beta_{1}}\right) .
$$

In particular, if a diagonal cycle $\beta_{1} \beta_{2} \cdots \beta_{K} \beta_{1}$ exists and $m \geq 2$ such that

$$
\rho\left(S_{m ; \beta_{1} \beta_{2}} S_{m ; \beta_{2} \beta_{3}} \cdots S_{m ; \beta_{K} \beta_{1}}\right)>1,
$$

or

$$
\rho\left(W_{m ; \beta_{1} \beta_{2}} W_{m ; \beta_{2} \beta_{3}} \cdots W_{m ; \beta_{K} \beta_{1}}\right)>1
$$

then $h\left(\mathbb{A}_{2}\right)>0$.

Proof. First, show that

$$
h\left(\mathbb{A}_{2}\right) \geq \frac{1}{m K} \limsup _{n \rightarrow \infty}\left(\log \rho\left(\left|\left(S_{m ; \beta_{1} \beta_{2}} S_{m ; \beta_{2} \beta_{3}} \cdots S_{m ; \beta_{K} \beta_{1}}\right)^{n} X_{m, 2 ; \beta_{1}}\right|\right) .\right.
$$

Indeed, from (1.11) and (2.71),

$$
\begin{aligned}
& h\left(\mathbb{A}_{2}\right)=\lim _{n \rightarrow \infty} \frac{1}{n K+2} \log \rho\left(\mathbb{A}_{n K+2}\right) \\
& =\lim _{n \rightarrow \infty} \frac{1}{m(n K+2)} \log \rho\left(\mathbb{A}_{n K+2}^{m}\right) \\
& \geq \frac{1}{m K} \limsup _{n \rightarrow \infty} \frac{1}{n}\left(\log \rho\left(\left|\left(S_{m ; \beta_{1} \beta_{2}} \cdots S_{m ; \beta_{K} \beta_{1}}\right)^{n} X_{m, 2 ; \beta_{1}}\right|\right)\right) .
\end{aligned}
$$


Now, the following remains to be shown

$$
\limsup _{n \rightarrow \infty} \frac{1}{n}\left(\log \rho\left(\left|\left(S_{m ; \beta_{1} \beta_{2}} \cdots S_{m ; \beta_{K} \beta_{1}}\right)^{n} X_{m, 2 ; \beta_{1}}\right|\right)=\log \rho\left(S_{m ; \beta_{1} \beta_{2}} \cdots S_{m ; \beta_{K} \beta_{1}}\right) .\right.
$$

Since $X_{m, 2 ; \beta_{1}}=\left(A_{m, 2 ; \beta_{1}}^{(k)}\right)^{t}$, if $\operatorname{tr}\left(A_{m, 2 ; \beta_{1}}^{(k)}\right)=0$ then Lemma 2.11 implies the $k$-th row of $S_{m ; \beta_{1} \beta_{2}}$ is zero which implies that the $k$-th row of $\left(S_{m ; \beta_{1} \beta_{2}} \cdots S_{m ; \beta_{K} \beta_{1}}\right)^{n}$ is also zero for any $n \geq 1$.

If $\operatorname{tr}\left(A_{m, 2 ; \beta_{1}}^{(k)}\right)=0$ for all $1 \leq k \leq 2^{m-1}$, then $S_{m ; \beta_{1} \beta_{2}} \equiv 0$. (2.80) holds trivially.

Now, assume that $1 \leq k^{\prime} \leq 2^{m-1}$ exists such that $\operatorname{tr}\left(A_{m, 2 ; \beta_{1}}^{\left(k^{\prime}\right)}>0\right.$. Define

$$
\hat{X}=\left(A_{m, 2 ; \beta_{1}}^{\left(k^{\prime}\right)}\right)^{t}=\left(\hat{X}_{1}, \cdots, \hat{X}_{M}\right)
$$

where $\operatorname{tr}\left(A_{m, 2 ; \beta_{1}}^{\left(k^{\prime}\right)}\right)>0$ for $1 \leq k^{\prime} \leq M \leq 2^{m-1}$. Then $\rho\left(\hat{X}_{j}\right)>0$ for $1 \leq j \leq M$.

Let $\mathbb{M}$ be the $M \times M$ sub-matrix of $S_{m ; \beta_{1} \beta_{2}} \cdots S_{m ; \beta_{K} \beta_{1}}$ from which the $k$-th row and $k$-th column have been removed whenever $\operatorname{tr}\left(A_{m, 2 ; \beta_{1}}^{(k)}\right)=0$ for $1 \leq k \leq 2^{m-1}$.

Clearly,

$$
\left|\left(S_{m ; \beta_{1} \beta_{2}} \cdots S_{m ; \beta_{K} \beta_{1}}\right)^{n} X_{m, 2 ; \beta_{1}}\right|=\left|\mathbb{M}^{n} \hat{X}\right|
$$

and

$$
\rho\left(S_{m ; \beta_{1} \beta_{2}} \cdots S_{m ; \beta_{K} \beta_{1}}\right)=\rho(\mathbb{M}) .
$$

The proof of (2.80) comprise three steps, according to

(i) $\mathbb{M}$ is primitive,

(ii) $\mathbb{M}$ is irreducible, and

(iii) $\mathbb{M}$ is reducible.

(i) $\mathbb{M}$ is primitive. Then by Perron-Frobenius Theorem the maximum eigenvalue $\rho(\mathbb{M})$ of $\mathbb{M}$ is unique with maximum modulus, i.e.

$$
\rho(\mathbb{M})=\lambda_{1}>\left|\lambda_{j}\right|,
$$

for all $2 \leq j \leq M$, where $\lambda_{j}$ are eigenvalues of $\mathbb{M}$. Moreover, a positive eigenvector $\mathbf{v}_{1}=\left(v_{1}, v_{2}, \cdots, v_{M}\right)^{t}$ is associated with $\lambda_{1}$ [26], [27]. Furthermore, Jordan canonical form theorem states that a non-singular matrix $\mathbb{P}=\left[P_{i j}\right]_{M \times M}$ exists, such that the real Jordan canonical form of $\mathbb{M}$ is

$$
\hat{\mathbb{M}} \equiv \mathbb{P M P}^{-1}=\left[\begin{array}{cccc}
\lambda_{1} & 0 & \cdots & 0 \\
0 & J_{n_{2}} & \cdots & 0 \\
\vdots & \vdots & \ddots & \vdots \\
0 & \cdots & \cdots & J_{n_{q}}
\end{array}\right]
$$

where $J_{n_{k}}, 2 \leq k \leq q$ are real Jordan blocks and the associated eigenvalue $\lambda_{k}$ of $J_{n_{k}}$ satisfies (2.84). Moreover, the positivity of eigenvector $\mathbf{v}_{1}$ implies that $\mathbb{P}$ can be chosen such that

$$
\sum_{i=1}^{M} P_{i j}=1
$$


and

$$
P_{1 j}>0
$$

for all $1 \leq j \leq M$. Therefore, by (2.86)

$$
\begin{aligned}
& \left|\mathbb{M}^{n} \hat{X}\right|=\left|\mathbb{P M}^{n} \hat{X}\right|=\left|\mathbb{P M}^{n} \mathbb{P}^{-1} \mathbb{P} \hat{X}\right| \\
& =\left|\left(\mathbb{P M} \mathbb{P}^{-1}\right)^{n} \mathbb{P} \hat{X}\right|=\left|\hat{\mathbb{M}}^{n} \mathbb{P} \hat{X}\right| \\
& =\lambda_{1}{ }^{n}\left\{\sum_{j=1}^{M} P_{1 j} \hat{X}_{j}+\sum_{j=1}^{M} q_{n, j} \hat{X}_{j}\right\}
\end{aligned}
$$

where

$$
\lim _{n \rightarrow \infty} q_{n, j}=0
$$

for all $1 \leq j \leq M$, by (2.84).

Hence, by (2.87) and (2.88),

$$
\lim _{n \rightarrow \infty} \frac{1}{n} \log \rho\left(\left|\mathbb{M}^{n} \hat{X}\right|\right)=\log \lambda_{1}
$$

Combining with (2.82), (2.83) and (2.89), (2.80) follows.

(ii) $\mathbb{M}$ is irreducible.

If $\mathbb{M}$ is irreducible but imprimitive, then $k \geq 2$ exists, such that

$$
\lambda_{1}=\left|\lambda_{2}\right|=\cdots=\left|\lambda_{k}\right|>\left|\lambda_{j}\right|
$$

for all $j>k$. Then, by applying a permutation, $\mathbb{M}$ can be expressed as

$$
\mathbb{M}=\left[\begin{array}{ccccc}
0 & M_{12} & 0 & \cdots & 0 \\
0 & 0 & M_{23} & \cdots & 0 \\
\vdots & \vdots & \vdots & \ddots & \vdots \\
0 & \vdots & \vdots & 0 & M_{k-1, k} \\
M_{k 1} & 0 & \cdots & \cdots & 0
\end{array}\right]
$$

and,

$$
\mathbb{M}^{k}=\left[\begin{array}{cccc}
M_{1} & 0 & \cdots & 0 \\
0 & M_{2} & \cdots & 0 \\
\vdots & \vdots & \ddots & \vdots \\
0 & \cdots & 0 & M_{k}
\end{array}\right]
$$

where $M_{j}=M_{j, j+1} M_{j+1, j+2} \cdots M_{j-1, j}$ is primitive with the maximum eigenvalue $\lambda_{1}^{k}$, see [26], [27]. Hence, by the same argument as in (i)

$$
\lim _{n \rightarrow \infty} \frac{1}{n} \log \rho\left(\left|\mathbb{M}^{n k} \hat{X}\right|\right)=\lambda_{1}^{k},
$$

(2.80) follows. 
(iii) $\mathbb{M}$ is reducible.

In this case, by applying a permutation, $\mathbb{M}$ can be expressed as a block upper triangular matrix:

$$
\mathbb{M}=\left[\begin{array}{ccccc}
M_{11} & M_{12} & \ldots & \ldots & M_{1 k} \\
0 & M_{22} & \cdots & \cdots & M_{2 k} \\
0 & 0 & \ldots & \ddots & \ldots \\
0 & 0 & \cdots & 0 & M_{k k}
\end{array}\right]
$$

where $M_{i i}$ is either irreducible or zero. Furthermore,

$$
\sigma(\mathbb{M})=\bigcup_{j=1}^{k} \sigma\left(\mathbb{M}_{j j}\right),
$$

where $\sigma(\mathbb{M})$ and $\sigma\left(\mathbb{M}_{j j}\right)$ are the sets of eigenvalues of $\mathbb{M}$ and $\mathbb{M}_{j j}$, respectively. In particular, $1 \leq j \leq k$ exists, such that

$$
\rho\left(\mathbb{M}_{j j}\right)=\rho(\mathbb{M})=\lambda_{1} .
$$

[26], [27]. Therefore, applying (2.83), (2.93) and the same argument as in (ii) yields (2.80).

The proof is complete.

Definition 2.13. Let $\mathcal{D}$ denote the set of all diagonal cycle:

$$
\mathcal{D}=\left\{\beta_{1} \beta_{2} \cdots \beta_{K} \beta_{K+1} \mid \beta_{1} \beta_{2} \cdots \beta_{K} \beta_{K+1} \text { satisfies (2.67) and (2.68) }\right\},
$$

define

$$
h_{*}\left(\mathbb{A}_{2}\right)=\sup _{m \geq 2, \beta_{1} \beta_{2} \cdots \beta_{K+1} \in \mathcal{D}} \frac{1}{m K} \log \rho\left(S_{m ; \beta_{1} \beta_{2}} S_{m ; \beta_{2} \beta_{3}} \cdots S_{m ; \beta_{K} \beta_{1}}\right) .
$$

and

$$
h_{*}^{\prime}\left(\mathbb{A}_{2}\right)=\sup _{m \geq 2, \beta_{1} \cdots \beta_{K} \in D} \frac{1}{m K} \log \rho\left(W_{m ; \beta_{1} \beta_{2}} W_{m ; \beta_{2} \beta_{3}} \cdots W_{m ; \beta_{K} \beta_{1}}\right) .
$$

Then Theorem 2.12 implies

$$
h\left(\mathbb{A}_{2}\right) \geq h_{*}\left(\mathbb{A}_{2}\right) \text { and } h\left(\mathbb{A}_{2}\right) \geq h_{*}^{\prime}\left(\mathbb{A}_{2}\right) .
$$

Knowing whether the equality holds for $\mathbb{A}_{2}$ is of interest, since $h_{*}\left(\mathbb{A}_{2}\right)$ and $h_{*}^{\prime}\left(\mathbb{A}_{2}\right)$ are more manageable than $h\left(\mathbb{A}_{2}\right)$. However, a class of $\mathbb{A}_{2}$ has been found for what equality (2.96) holds; details can be found in Example 2.14. of the next subsection.

2.3. Examples of transition matrices with positive entropy. In this subsection, various examples are studied to elucidate the power of Theorem 2.12 in verifying that the entropies are positive. First, Golden-Mean type transition matrices are studied. 
Example 2.14.

(A) Golden-Mean

When two symbols on two-cell horizontal lattice $\mathbb{Z}_{2 \times 1}$ and vertical lattice $\mathbb{Z}_{1 \times 2}$ are considered and both transition matrices are given by golden-mean, i.e.,

$$
\mathbb{H}_{1}=\mathbb{V}_{1}=\left[\begin{array}{ll}
1 & 1 \\
1 & 0
\end{array}\right]
$$

then the (horizontal) transition matrix $\mathbb{A}_{2}$ on $\mathbb{Z}_{2 \times 2}$ is

$$
\mathbb{A}_{2}=\left[\begin{array}{llll}
1 & 1 & 1 & 0 \\
1 & 0 & 1 & 0 \\
1 & 1 & 0 & 0 \\
0 & 0 & 0 & 0
\end{array}\right]
$$

as in [41]. Verifying

$$
\mathbb{B}_{2}=\widetilde{\mathbb{A}}_{2}=\widetilde{\mathbb{B}}_{2}=\mathbb{A}_{2} .
$$

is also easy. Furthermore, for any $n \geq 2$,

$$
\mathbb{A}_{n+1}=\left[\begin{array}{cc}
A_{n+1} & B_{n+1} \\
C_{n+1} & 0
\end{array}\right]=\left[\begin{array}{cccc}
A_{n} & B_{n} & A_{n} & 0 \\
C_{n} & 0 & C_{n} & 0 \\
A_{n} & B_{n} & 0 & 0 \\
0 & 0 & 0 & 0
\end{array}\right]
$$

where

$$
A_{n+1}=\left[\begin{array}{cc}
A_{n} & B_{n} \\
C_{n} & 0
\end{array}\right]
$$

with $C_{n}=B_{n}{ }^{t}$ and $A_{n}{ }^{t}=A_{n}$, i.e., $\mathbb{A}_{n}$ are symmetric for all $n \geq 2$.

Moreover, the following two properties hold:

(i) For any $m \geq 2$,

$$
C_{m ; 11}=\mathbb{A}_{m-1},
$$

where

$$
\mathbb{A}_{1} \equiv\left[\begin{array}{ll}
a_{11} a_{11} & a_{12} a_{21} \\
a_{13} a_{31} & a_{14} a_{41}
\end{array}\right]
$$

and

(ii) for any $m \geq 2$,

$$
\frac{1}{m} \log \rho\left(\mathbb{A}_{m-1}\right) \leq h\left(\mathbb{A}_{2}\right) \leq \frac{1}{m} \log \rho\left(\mathbb{A}_{m}\right) .
$$

Therefore,

$$
h\left(\mathbb{A}_{2}\right)=h_{*}\left(\mathbb{A}_{2}\right)>0 .
$$

The numerical results appears in Example 3.12. 
(B) Simplified Golden-Mean.

Consider

$$
\mathbb{A}_{2}=\left[\begin{array}{llll}
1 & 1 & 1 & 0 \\
1 & 0 & 0 & 0 \\
1 & 0 & 0 & 0 \\
0 & 0 & 0 & 0
\end{array}\right]
$$

(2.104) cannot be generated from one-dimensional transition matrices $\mathbb{H}_{1}$ and $\mathbb{V}_{1}$, as in the Golden-Mean (2.97). Equation (2.104) is obtained by letting $a_{23}=a_{32}=0$ in the Golden-Mean (2.97). (2.98) is easily verified, and for any $n \geq 2$,

$$
\mathbb{A}_{n+1}=\left[\begin{array}{cccc} 
& & \mathbb{A}_{n-1} & 0 \\
\mathbb{A}_{n} & & 0 & 0 \\
\mathbb{A}_{n-1} & 0 & 0 & 0 \\
0 & 0 & 0 & 0
\end{array}\right] .
$$

Furthermore, (i), (ii) and (2.103) hold as in (A).

(C) Generally, if $\mathbb{A}_{2}$ satisfies the following three conditions

(C1) $\mathbb{B}_{2}=\mathbb{A}_{2}$,

(C2) $a_{1 j}=1$ if $A_{2 ; j} \neq 0$ for $1 \leq j \leq 4$,

(C3) $\widetilde{A}_{2 ; 1} \geq A_{2 ; j}$ for $1 \leq j \leq 4$,

then (i), (ii) and (2.103) hold. The matrices $\mathbb{A}_{2}$, which satisfy $(\mathrm{C} 1),(\mathrm{C} 2)$ and (C3) can be listed as

$$
\left[\begin{array}{cccc}
1 & 1 & 1 & 0 \\
1 & 0 & a_{23} & 0 \\
1 & a_{32} & 0 & 0 \\
0 & 0 & 0 & 0
\end{array}\right]
$$

and

$$
\left[\begin{array}{cccc}
1 & 1 & 1 & 1 \\
1 & 1 & a_{23} & a_{24} \\
1 & a_{32} & 1 & a_{34} \\
1 & a_{34} & a_{43} & a_{44}
\end{array}\right]
$$

where $a_{i j}$ is either 0 or 1 in (2.106) and (2.107).

Notably, if (C2) and (C3) are replaced by

$(\mathrm{C} 2)^{\prime} a_{4 j}=1$ if $A_{2 ; j} \neq 0$ for $1 \leq j \leq 4$,

(C3) $\widetilde{A}_{2 ; 4} \geq A_{2 ; j}$ for $1 \leq j \leq 4$,

then for any $m \geq 2$,

$$
C_{m ; 44}=\mathbb{A}_{m-1}
$$

with

$$
\mathbb{A}_{1}=\left[\begin{array}{ll}
a_{41} a_{14} & a_{42} a_{24} \\
a_{43} a_{34} & a_{44} a_{44}
\end{array}\right],
$$

and property (ii) and equation (2.103) hold. 
In Example 2.14, the diagonal parts $A_{2 ; 1}$ or $A_{2 ; 4}$ are dominant. In this case, only $C_{m ; 11}$ or $C_{m ; 44}$ is required to apply Theorem 2.12. In contrast, when $\mathbb{A}_{2 ; 1}$ and $\mathbb{A}_{2 ; 4}$ are no longer dominant as in the following examples, $A_{2 ; 2}$ and $A_{2 ; 3}$ can complement each other to establish that the entropy is positive.

Example 2.15.

(A) Consider

$$
\mathbb{A}_{2}=\left[\begin{array}{llll}
0 & 1 & 1 & 0 \\
1 & 0 & 1 & 0 \\
1 & 1 & 0 & 0 \\
0 & 0 & 0 & 0
\end{array}\right],
$$

that (2.98) holds can be verified and

$$
\begin{aligned}
& C_{2 ; 11}=\left[\begin{array}{cc}
0 & 1 \\
1 & 0 \\
1 & 1 \\
0 & 0
\end{array}\right], \quad C_{2 ; 22}=\left[\begin{array}{cc}
1 & 0 \\
1 & 0 \\
0 & 0 \\
0 & 0
\end{array}\right] \\
& C_{2 ; 33}=
\end{aligned}
$$

Therefore,

$$
S_{2 ; 14} S_{2 ; 41}=\left[\begin{array}{cc}
1 & 1 \\
1 & 1
\end{array}\right]
$$

and

$$
h\left(\mathbb{A}_{2}\right) \geq \frac{1}{4} \log 2 .
$$

(B) Consider

$$
\mathbb{A}_{2}=\left[\begin{array}{llll}
0 & 1 & 1 & 0 \\
1 & 0 & 1 & 1 \\
1 & 0 & 0 & 1 \\
1 & 1 & 1 & 0
\end{array}\right]
$$

Then verifying

$$
\mathbb{B}_{2}=\left[\begin{array}{llll}
0 & 1 & 1 & 0 \\
1 & 0 & 1 & 1 \\
1 & 0 & 1 & 1 \\
0 & 1 & 1 & 0
\end{array}\right], \quad \widetilde{\mathbb{B}}_{2}=\left[\begin{array}{llll}
0 & 1 & 1 & 0 \\
1 & 0 & 0 & 1 \\
1 & 1 & 0 & 1 \\
1 & 1 & 1 & 0
\end{array}\right], \quad \text { and } \widetilde{\mathbb{A}}_{2}=\left[\begin{array}{llll}
0 & 1 & 1 & 0 \\
1 & 1 & 0 & 1 \\
1 & 1 & 0 & 1 \\
0 & 1 & 1 & 0
\end{array}\right]
$$

is simple.

Furthermore,

$$
\begin{aligned}
& C_{2 ; 11}=\left[\begin{array}{cc}
0 & 1 \\
1 & 0 \\
1 & 0 \\
0 & 1
\end{array}\right], \quad C_{2 ; 22}=\left[\begin{array}{cc}
1 & 0 \\
0 & 1 \\
0 & 1 \\
1 & 0
\end{array}\right] \\
& C_{2 ; 33}=
\end{aligned}
$$

and

$$
\begin{aligned}
& U_{2 ; 11}=\left[\begin{array}{cc}
0 & 1 \\
1 & 0 \\
1 & 0 \\
1 & 1
\end{array}\right], \quad U_{2 ; 22}=\left[\begin{array}{cc}
1 & 0 \\
0 & 1 \\
U_{2 ; 33} & 1 \\
1 & 0
\end{array}\right] .
\end{aligned}
$$


Now, for any diagonal cycle, $\beta_{1} \cdots \beta_{K} \beta_{1}, \rho\left(S_{2 ; \beta_{1} \beta_{2}} \cdots S_{2 ; \beta_{K} \beta_{1}}\right)=1, h\left(\mathbb{A}_{2}\right)>0$ cannot be established.

However,

$$
W_{2 ; 11} W_{2 ; 14} W_{2 ; 41}=U_{2 ; 11} U_{2 ; 22} U_{2 ; 33}=\left[\begin{array}{cc}
1 & 1 \\
1 & 0
\end{array}\right]
$$

which implies

$$
h\left(\mathbb{A}_{2}\right) \geq \frac{1}{6} \log g
$$

where

$$
g=\frac{1}{2}(1+\sqrt{5})
$$

is the golden mean, which is a root of $\lambda^{2}-\lambda-1=0$.

This example demonstrates the asymmetry of $\mathbb{A}_{2}$ and $\mathbb{B}_{2}$ in applying Theorem 2.12 , to verify the entropy is positive. Both $\mathbb{C}_{m}$ and $\mathbb{U}_{m}$ are typically checked for completeness.

Example 2.16. Consider

$$
\mathbb{A}_{2}=\left[\begin{array}{llll}
1 & 1 & 1 & 1 \\
0 & 0 & 0 & 1 \\
0 & 0 & 0 & 1 \\
1 & 0 & 0 & 0
\end{array}\right]
$$

Then it is easy to check that

$$
W_{2 ; 11} W_{2 ; 14} W_{2 ; 41}=\left[\begin{array}{cc}
2 & 0 \\
0 & 0
\end{array}\right], \quad S_{3 ; 44}=\left[\begin{array}{cc}
G & 0 \\
0 & 0
\end{array}\right],
$$

and

$$
S_{4 ; 44}=\left[\begin{array}{cccc}
G & 0 & 0 & 0 \\
0 & e_{1} & 0 & 0 \\
0 & 0 & 0 & 0 \\
0 & 0 & 0 & 0
\end{array}\right]
$$

where

$$
G=\left[\begin{array}{ll}
1 & 1 \\
1 & 0
\end{array}\right] \text { and } e_{1}=\left[\begin{array}{ll}
1 & 0 \\
0 & 0
\end{array}\right]
$$

Therefore,

$$
h\left(\mathbb{A}_{2}\right) \geq \max \left\{\frac{1}{6} \log 2, \frac{1}{3} \log g, \frac{1}{4} \log g\right\}=\frac{1}{3} \log g .
$$

Example 2.17. Consider

$$
\mathbb{A}_{2}=\left[\begin{array}{llll}
0 & 1 & 1 & 1 \\
1 & 0 & 0 & 0 \\
1 & 0 & 0 & 0 \\
1 & 0 & 0 & 0
\end{array}\right]
$$


Then

$$
\mathbb{B}_{2}=\left[\begin{array}{llll}
0 & 1 & 1 & 0 \\
1 & 1 & 0 & 0 \\
1 & 0 & 1 & 0 \\
0 & 0 & 0 & 0
\end{array}\right]=\tilde{\mathbb{A}}_{2} \text { and } \tilde{\mathbb{B}}_{2}=\mathbb{A}_{2}
$$

Therefore

$$
C_{2,11}=\left[\begin{array}{ll}
0 & 1 \\
1 & 1
\end{array}\right] \equiv G^{\prime}
$$

Furthermore,

$$
C_{4 ; 11}=G^{\prime} \otimes e_{1} \otimes G^{\prime}
$$

and

$$
C_{2 m ; 11}=G^{\prime} \otimes\left(\otimes\left(e_{1} \otimes G^{\prime}\right)^{m-1}\right)
$$

can be proved, and which implies

$$
\frac{1}{2 m} \log \rho\left(C_{2 m ; 11}\right)=\frac{1}{2} \log g .
$$

for all $m \geq 1$. Hence, $h\left(\mathbb{A}_{2}\right) \geq \frac{1}{2} \log g$. Moreover, in Remark 3.10 (ii), it can be shown that $h\left(\mathbb{A}_{2}\right)=\frac{1}{2} \log g$

\section{Trace operators.}

3.1. Trace operator $\mathbb{T}_{m}$. The preceding section introduces connecting operators $\mathbb{C}_{m}$, which can be used to find lower bounds of spatial entropy. This section studies the diagonal part of $\mathbb{C}_{m}$, which can be used to investigate the trace of $\mathbb{A}_{n}^{m}$. When $\mathbb{A}_{2}$ is symmetric, $\mathbb{T}_{2 m}$ gives the upper bound of spatial entropy.

The trace operator is defined first.

Definition 3.1. For $m \geq 2$, the $m$-th order trace operator $\mathbb{T}_{m}$ of $\mathbb{A}_{2}$ is defined by

$$
\mathbb{T}_{m}=\left[\begin{array}{ll}
C_{m ; 11} & C_{m ; 22} \\
C_{m ; 33} & C_{m ; 44}
\end{array}\right]=\left[\begin{array}{ll}
S_{m ; 11} & S_{m ; 14} \\
S_{m ; 41} & S_{m ; 44}
\end{array}\right]
$$

where $C_{m ; i j}$ is as given in (1.23) or (2.29).

Similarly, the $m$-th order trace operator $\mathbb{T}_{m}^{\prime}$ of $\mathbb{B}_{2}$ is defined by

$$
\mathbb{T}_{m}^{\prime}=\left[\begin{array}{ll}
U_{m ; 11} & U_{m ; 22} \\
U_{m ; 33} & U_{m ; 44}
\end{array}\right]=\left[\begin{array}{ll}
W_{m ; 11} & W_{m ; 14} \\
W_{m ; 41} & W_{m ; 44}
\end{array}\right]
$$

where $U_{m ; i j}$ is as given in (2.31). follows.

The relationships between the trace operator $\mathbb{T}_{m}, \mathbb{T}_{m}^{\prime}$ and $\mathbb{A}_{m}, \mathbb{B}_{m}$ are given as 
TheOREM 3.2. For any $m \geq 2$,

$$
\mathbb{T}_{m}=\left(\mathbb{B}_{m}\right)_{2^{m} \times 2^{m}} \circ\left[\begin{array}{ll}
E_{2^{m-2} \times 2^{m-2}} \otimes\left[\begin{array}{ll}
a_{11} & a_{21} \\
a_{31} & a_{41}
\end{array}\right] & E_{2^{m-2} \times 2^{m-2}} \otimes\left[\begin{array}{ll}
a_{12} & a_{22} \\
a_{32} & a_{42}
\end{array}\right] \\
E_{2^{m-2} \times 2^{m-2}} \otimes\left[\begin{array}{ll}
a_{13} & a_{23} \\
a_{33} & a_{43}
\end{array}\right] & E_{2^{m-2} \times 2^{m-2}} \otimes\left[\begin{array}{ll}
a_{14} & a_{24} \\
a_{34} & a_{44}
\end{array}\right]
\end{array}\right]
$$

and

$$
\mathbb{T}_{m}^{\prime}=\left(\mathbb{A}_{m}\right)_{2^{m} \times 2^{m}} \circ\left[\begin{array}{ll}
E_{2^{m-2} \times 2^{m-2}} \otimes\left[\begin{array}{ll}
b_{11} & b_{21} \\
b_{31} & b_{41}
\end{array}\right] & E_{2^{m-2} \times 2^{m-2}} \otimes\left[\begin{array}{ll}
b_{12} & b_{22} \\
b_{32} & b_{42}
\end{array}\right] \\
E_{2^{m-2} \times 2^{m-2}} \otimes\left[\begin{array}{ll}
b_{13} & b_{23} \\
b_{33} & b_{43}
\end{array}\right] & E_{2^{m-2} \times 2^{m-2}} \otimes\left[\begin{array}{ll}
b_{14} & b_{24} \\
b_{34} & b_{44}
\end{array}\right]
\end{array}\right] .
$$

In particular,

$$
\mathbb{T}_{m} \leq \mathbb{B}_{m} \text { and } \mathbb{T}_{m}^{\prime} \leq \mathbb{A}_{m}
$$

Proof. By (3.1) and (2.29),

$\mathbb{T}_{m}=\left(\mathbb{B}_{m}\right)_{2^{m} \times 2^{m}} \circ\left[\begin{array}{cc}E_{2^{m-2} \times 2^{m-2}} \otimes\left[\begin{array}{ll}a_{11} & a_{21} \\ a_{31} & a_{41}\end{array}\right] & E_{2^{m-2} \times 2^{m-2}} \otimes\left[\begin{array}{ll}a_{12} & a_{22} \\ a_{32} & a_{42}\end{array}\right] \\ E_{2^{m-2} \times 2^{m-2}} \otimes\left[\begin{array}{ll}a_{13} & a_{23} \\ a_{33} & a_{43}\end{array}\right] & E_{2^{m-2} \times 2^{m-2}} \otimes\left[\begin{array}{ll}a_{14} & a_{24} \\ a_{34} & a_{44}\end{array}\right]\end{array}\right]$.

A similar result also holds for $\mathbb{T}_{m}^{\prime}$. Hence, (3.5) follows immediately.

The proof is complete.

Notably, the trace operator $\mathbb{T}_{m}$ (or $\mathbb{T}_{m}^{\prime}$ ) preserves all periodic words $a_{i_{1} i_{2}} a_{i_{2} i_{3}} \cdots a_{i_{m} i_{m+1}}\left(b_{i_{1} i_{2}} b_{i_{2} i_{3}} \cdots b_{i_{m} i_{m+1}}\right)$ with $i_{m+1}=i_{1}$ of length $m$ systematically as $\mathbb{B}_{m}$ (or $\left.\mathbb{A}_{m}\right)$.

The traces of the elementary patterns are defined accordingly.

Definition 3.3. For $m, n \geq 2$ and $1 \leq \alpha \leq 4$, define

$$
\begin{gathered}
t_{m, n ; \alpha}^{(k)}=\operatorname{tr}\left(A_{m, n ; \alpha}^{(k)}\right), \\
\operatorname{tr}\left(X_{m, n ; \alpha}\right)=\left(t_{m, n ; \alpha}^{(k)}\right)_{1 \leq k \leq 2^{m-1}},
\end{gathered}
$$

and

$$
t_{m, n}=\left(\operatorname{tr}\left(X_{m, n ; 1}\right), \operatorname{tr}\left(X_{m, n ; 4}\right)\right)^{t},
$$

which are $2^{m-1}$ and $2^{m}$ vectors, respectively.

Note that

$$
\begin{aligned}
\operatorname{tr}\left(\mathbb{A}_{n}^{m}\right) & =\operatorname{tr}\left(\sum_{k=1}^{2^{m-1}} A_{m, n ; 1}^{(k)}+\sum_{k=1}^{2^{m-1}} A_{m, n ; 4}^{(k)}\right) \\
& =\left|\operatorname{tr}\left(X_{m, n ; 1}\right)\right|+\left|\operatorname{tr}\left(X_{m, n ; 4}\right)\right| \\
& =\left|t_{m, n}\right| .
\end{aligned}
$$


First prove that $\mathbb{T}_{m}$ can reduce the traces of higher-order to lower-order.

Proposition 3.4. For $m \geq 2$ and $n \geq 2$,

$$
t_{m, n+1}=\mathbb{T}_{m} t_{m, n}
$$

Proof. By Theorem 2.5, it is easy to see

$$
\left(\begin{array}{c}
\operatorname{tr}\left(X_{m, n+1 ; 1}\right) \\
\operatorname{tr}\left(X_{m, n+1 ; 4}\right)
\end{array}\right)=\left(\begin{array}{c}
C_{m ; 11} \operatorname{tr}\left(X_{m, n ; 1}\right)+C_{m ; 22} \operatorname{tr}\left(X_{m, n ; 4}\right) \\
C_{m ; 33} \operatorname{tr}\left(X_{m, n ; 1}\right)+C_{m ; 44} \operatorname{tr}\left(X_{m, n ; 4}\right)
\end{array}\right) .
$$

Then, (3.10) follows immediately.

The proof is complete.

Repeatedly applying Proposition 3.4 yields the following result.

TheOREM 3.5. For $m \geq 2$ and $n \geq 1$,

$$
\begin{gathered}
\operatorname{tr}\left(\mathbb{A}_{n+2}^{m}\right)=\left|\mathbb{T}_{m}^{n} t_{m, 2}\right| \\
\equiv \sum_{\beta_{k} \in\{1,4\}}\left|S_{m ; \beta_{1} \beta_{2}} S_{m ; \beta_{2} \beta_{3}} \cdots S_{m ; \beta_{n} \beta_{n+1}} \operatorname{tr}\left(X_{m, 2 ; \beta_{n+1}}\right)\right| .
\end{gathered}
$$

Proof.

$$
\begin{aligned}
& \operatorname{tr}\left(\mathbb{A}_{n}^{m}\right) \\
= & \sum_{k=1}^{2^{m-1}} \operatorname{tr}\left(A_{m, n ; 1 ; 1}^{(k)}\right)+\sum_{k=1}^{2^{m-1}} \operatorname{tr}\left(A_{m, n ; 1 ; 4}^{(k)}\right)+\sum_{k=1}^{2^{m-1}} \operatorname{tr}\left(A_{m, n ; 4 ; 1}^{(k)}\right)+\sum_{k=1}^{2^{m-1}} \operatorname{tr}\left(A_{m, n ; 4 ; 4}^{(k)}\right) \\
= & \left|\operatorname{tr}\left(X_{m, n ; 1 ; 1}\right)\right|+\left|\operatorname{tr}\left(X_{m, n ; 1 ; 4}\right)\right|+\left|\operatorname{tr}\left(X_{m, n ; 4 ; 1}\right)\right|+\left|\operatorname{tr}\left(X_{m, n ; 4 ; 4}\right)\right| \\
= & \left|\operatorname{tr}\left(S_{m ; 11} X_{m, n-1 ; 1}\right)\right|+\left|\operatorname{tr}\left(S_{m ; 14} X_{m, n-1 ; 4}\right)\right| \\
& +\left|\operatorname{tr}\left(S_{m ; 41} X_{m, n-1 ; 1}\right)\right|+\left|\operatorname{tr}\left(S_{m ; 44} X_{m, n-1 ; 4}\right)\right| \\
= & \left|\mathbb{T}_{m} t_{m, n-1}\right|,
\end{aligned}
$$

here Theorem 2.4 is used.

Reduction on $n$, yields

$$
\operatorname{tr}\left(\mathbb{A}_{n}^{m}\right)=\left|\mathbb{T}_{m}^{n-2} t_{m, 2}\right|
$$

Finally, (3.12) follows from (3.1) and (3.8).

The proof is complete.

The following lemma is needed to show (1.33).

Lemma 3.6. Let $V_{m}$ be a nonnegative eigenvector of $\mathbb{T}_{m}$ with respect to the maximum eigenvalue $\rho\left(\mathbb{T}_{m}\right)$. If $\rho\left(\mathbb{T}_{m}\right)>0$, then

$$
\left\langle V_{m}, t_{m, 2}\right\rangle>0,
$$

where $\langle$,$\rangle denotes the standard inner product of \mathbb{C}^{2^{m}}$.

Proof. Let $V_{m}=\left(u_{1}, \cdots, u_{M}, u_{1}^{\prime}, \cdots, u_{M}^{\prime}\right)$ be a nonnegative eigenvector of $\mathbb{T}_{m}$, where $M=2^{m-1}$. Since $\rho\left(\mathbb{T}_{m}\right)>0$, by Lemma 2.11, if $u_{k}>0$ (or $u_{l}^{\prime}>0$ ) then $\operatorname{tr}\left(A_{m, 2 ; 1}^{(k)}\right)>0$ (or $\left.\operatorname{tr}\left(A_{m, 2 ; 4}^{(l)}\right)>0\right)$. The result follows by (3.8). 
The proof is complete.

Now, (1.33) can be proved.

Theorem 3.7. For any $m \geq 2$,

$$
\limsup _{n \rightarrow \infty} \frac{1}{n} \log \operatorname{tr}\left(\mathbb{A}_{n}^{m}\right)=\log \rho\left(\mathbb{T}_{m}\right)
$$

and

$$
h\left(\mathbb{A}_{2}\right) \geq \limsup _{m \rightarrow \infty} \frac{1}{m} \log \rho\left(\mathbb{T}_{m}\right) .
$$

Furthermore, if $\mathbb{A}_{n}$ are primitive for all $n \geq 2$, then limsup in (3.13) and (3.14) can be replaced by lim, i.e.,

$$
\lim _{n \rightarrow \infty} \frac{1}{n} \log \operatorname{tr}\left(\mathbb{A}_{n}^{m}\right)=\log \rho\left(\mathbb{T}_{m}\right)
$$

and

$$
h\left(\mathbb{A}_{2}\right) \geq \lim _{m \rightarrow \infty} \frac{1}{m} \log \rho\left(\mathbb{T}_{m}\right) .
$$

Proof. By Perron-Frobenius theorem, for all $n \geq 2$, we have

$$
\limsup _{m \rightarrow \infty} \frac{1}{m} \log \operatorname{tr}\left(\mathbb{A}_{n}^{m}\right)=\log \rho\left(\mathbb{A}_{n}\right) .
$$

Therefore, by (3.17) and Theorem 3.5, we have

$$
h\left(\mathbb{A}_{2}\right)=\lim _{n \rightarrow \infty} \frac{1}{n} \log \rho\left(\mathbb{A}_{n}\right)=\limsup _{n, m \rightarrow \infty} \frac{1}{m n} \log \operatorname{tr}\left(\mathbb{A}_{n}^{m}\right)=\limsup _{n, m \rightarrow \infty} \frac{1}{m n} \log \left|\mathbb{T}_{m}^{n} t_{m, 2}\right| .
$$

By Lemma 3.6 and by argument used to prove Theorem 2.12,

$$
\limsup _{n \rightarrow \infty} \frac{1}{n} \log \left|\mathbb{T}_{m}^{n} t_{m, 2}\right|=\log \rho\left(\mathbb{T}_{m}\right)
$$

can be shown, and (3.13) and (3.14) follow immediately.

When $\mathbb{A}_{n}$ are primitive for all $n \geq 2,(3.15)$ and (3.16) follow.

The proof is complete.

Now, the symmetry of $\mathbb{A}_{2}$ is established to be able to be inherited by the higher order matrices.

Proposition 3.8. If $\mathbb{A}_{2}$ is symmetric, then $\mathbb{A}_{n}$ is also symmetric for each $n \geq 3$.

Proof. The proposition is proven by induction on $n$.

Let $\mathbb{M}=\left[\begin{array}{ll}M_{1} & M_{2} \\ M_{3} & M_{4}\end{array}\right]$ be a square matrix and $M_{i}, 1 \leq i \leq 4$, all be square matrices. Then, the transpose matrix $\mathbb{M}^{t}$ of $\mathbb{M}$ is

$$
\mathbb{M}^{t}=\left[\begin{array}{ll}
M_{1}^{t} & M_{3}{ }^{t} \\
M_{2}^{t} & M_{4}^{t}
\end{array}\right]
$$


Therefore, $\mathbb{M}$ is symmetric if and only if

$$
M_{1}^{t}=M_{1}, M_{3}^{t}=M_{2} \text { and } M_{4}^{t}=M_{4} .
$$

In particular, $\mathbb{A}_{2}$ is symmetric if and only if

$$
A_{2 ; 1}^{t}=A_{2 ; 1}, A_{2 ; 3}^{t}=A_{2 ; 2} \text { and } A_{2 ; 4}^{t}=A_{2 ; 4} .
$$

Now, $\mathbb{A}_{n}$ is assumed to be symmetric, such that

$$
A_{n ; 1}^{t}=A_{n ; 1}, A_{n ; 3}^{t}=A_{n ; 2} \text { and } A_{n ; 4}^{t}=A_{n ; 4} .
$$

Since

$$
A_{n+1 ; \alpha}=\left[A_{2 ; \alpha}\right]_{2 \times 2} \circ\left[\begin{array}{cc}
A_{n ; 1} & A_{n ; 2} \\
A_{n ; 3} & A_{n ; 4}
\end{array}\right],
$$

(3.19) and (3.20) imply

$$
A_{n+1 ; 1}^{t}=A_{n+1 ; 1}, A_{n+1 ; 3}^{t}=A_{n+1 ; 2} \text { and } A_{n+1 ; 4}^{t}=A_{n+1 ; 4} .
$$

Hence, $\mathbb{A}_{n+1}$ is symmetric.

The proof is complete.

Now, upper estimates of spatial entropy $h\left(\mathbb{A}_{2}\right)$ are obtained when $\mathbb{A}_{2}$ is symmetric.

Theorem 3.9. If $\mathbb{A}_{2}$ is symmetric then for any $m \geq 1$,

$$
h\left(\mathbb{A}_{2}\right) \leq \frac{1}{2 m} \log \rho\left(\mathbb{T}_{2 m}\right) .
$$

Proof. By Proposition 3.8, $\mathbb{A}_{n}^{2 m}$ is symmetric for any $m \geq 1$. The symmetry of $\mathbb{A}_{n}^{2 m}$ implies that all eigenvalues of $\mathbb{A}_{n}^{2 m}$ are non-negative. Hence,

$$
\rho\left(\mathbb{A}_{n}\right)^{2 m}=\rho\left(\mathbb{A}_{n}^{2 m}\right) \leq \operatorname{tr}\left(\mathbb{A}_{n}^{2 m}\right) .
$$

On the other hand, the subadditivity of (2.58) implies

$$
h\left(\mathbb{A}_{2}\right) \leq \frac{1}{(2 m k+1) n} \log \left|\mathbb{A}_{n}^{2 m k}\right| .
$$

Therefore, (3.22), (3.23) and (3.11) imply

$$
\begin{aligned}
h\left(\mathbb{A}_{2}\right) & \leq \lim _{n, k \rightarrow \infty} \frac{1}{(2 m k+1) n} \log \left|\mathbb{A}_{n}^{2 m k}\right|=\lim _{n \rightarrow \infty} \frac{1}{2 m n} \log \rho\left(\mathbb{A}_{n}^{2 m}\right) \\
& \leq \lim _{n \rightarrow \infty} \frac{1}{2 m n} \log \operatorname{tr}\left(\mathbb{A}_{n}^{2 m}\right)=\lim _{n \rightarrow \infty} \frac{1}{2 m n} \log \left|\mathbb{T}_{2 m}^{n-2} t_{2 m, 2}\right| \\
& \leq \frac{1}{2 m} \log \rho\left(\mathbb{T}_{2 m}\right) .
\end{aligned}
$$

The proof is complete.

Notably, $\mathbb{T}_{m}$ (or $\mathbb{T}_{m}^{\prime}$ ) yields a better estimate than $\mathbb{B}_{n}\left(\right.$ or $\mathbb{A}_{n}$ ) whenever

$$
h\left(\mathbb{A}_{2}\right) \leq \frac{1}{m} \log \rho\left(\mathbb{T}_{m}\right)
$$

holds. 
Remark 3.10.

(i) The problem in which $\mathbb{A}_{n}$ are primitive for all $n \geq 2$ has already been investigated [6]. In [6], various sufficient conditions have been found to ensure that $\mathbb{A}_{n}$ are primitive for all $n \geq 2$. Notably, limit in (3.15) and (3.16), instead of limsup in (3.13) and (3.14), causes $\mathbb{A}_{n}$ to have a unique maximum eigenvalue with a maximum modulus. Therefore, $\mathbb{A}_{n}$ may be imprimitive but (3.15) and (3.16) still hold. For example, Golden-Mean and simplified Golden-Mean in Example 2.14 are imprimitive but (3.15) and (3.16) still hold. The remaining matrices of these $\mathbb{A}_{n}$ are primitive if their rows and columns with zero entries are removed.

(ii) In general, limsup cannot be replaced by limit. For example, consider

$$
\mathbb{A}_{2}=\left[\begin{array}{llll}
0 & 1 & 1 & 1 \\
1 & 0 & 0 & 0 \\
1 & 0 & 0 & 0 \\
1 & 0 & 0 & 0
\end{array}\right]
$$

Further computation shows that

$$
\mathbb{T}_{2 m+1}=0
$$

and

$$
\mathbb{T}_{2 m}=\left[\begin{array}{cc}
\left(\otimes\left(G^{\prime} \otimes e_{1}\right)^{m-1}\right) \otimes G^{\prime} & e_{1} \otimes\left(\otimes\left(G^{\prime} \otimes e_{1}\right)^{m-1}\right) \\
e_{1} \otimes\left(\otimes\left(G^{\prime} \otimes e_{1}\right)^{m-1}\right) & e_{1} \otimes\left(\otimes\left(G^{\prime} \otimes e_{1}\right)^{m-1}\right)
\end{array}\right]
$$

for all $m \geq 1$, where $G^{\prime}=\left[\begin{array}{ll}0 & 1 \\ 1 & 1\end{array}\right]$ and $e_{1}=\left[\begin{array}{ll}1 & 0 \\ 0 & 0\end{array}\right]$.

Therefore, $\rho\left(\mathbb{T}_{2 m+1}\right)=0$. Furthermore, it can be shown that

$$
\rho\left(\mathbb{T}_{2 m}\right) \leq g^{m}+g^{m-1} .
$$

Combining (2.116) and (3.26), $h\left(\mathbb{A}_{2}\right)=\frac{1}{2} \log g$. Hence (3.14) holds only for limsup. Unlike (2.62) this example demonstrates that (3.24) does not hold for any $n=2 m+1$. This phenomenon is a disadvantage in determining the upper estimate of entropy associated with replacing $\mathbb{A}_{n}$ with $\mathbb{T}_{n}$.

Example 3.11. Consider

$$
\mathbb{A}_{2}=\left[\begin{array}{llll}
1 & 1 & 1 & 1 \\
0 & 0 & 0 & 1 \\
0 & 0 & 0 & 1 \\
1 & 0 & 0 & 0
\end{array}\right]
$$

which was studied as in Example 2.16. Now, $\mathbb{A}_{2}$ is asymmetric. Furthermore,

$$
\operatorname{tr}\left(\mathbb{A}_{n}^{2}\right)=3
$$

can be obtained for all $n \geq 2$. Hence, (3.22) and then (3.21) fail when $m=1$. However,

$$
C_{4 ; 44}=\left[\begin{array}{cccc}
G & 0 & 0 & 0 \\
0 & e_{1} & 0 & 0 \\
0 & 0 & 0 & 0 \\
0 & 0 & 0 & 0
\end{array}\right]
$$


where $G=\left[\begin{array}{ll}1 & 1 \\ 1 & 0\end{array}\right], e_{1}=\left[\begin{array}{ll}1 & 0 \\ 0 & 0\end{array}\right]$ and $0=\left[\begin{array}{ll}0 & 0 \\ 0 & 0\end{array}\right]$. Hence $\operatorname{tr}\left(\mathbb{A}_{n}^{4}\right)$ grows at least exponentially with exponent $\rho(G)=g$, the golden-mean.

Whether (3.21) holds for some $m \geq 2$ is of interest.

Example 3.12. Consider the Golden-Mean

$$
\mathbb{A}_{2}=\left[\begin{array}{llll}
1 & 1 & 1 & 0 \\
1 & 0 & 1 & 0 \\
1 & 1 & 0 & 0 \\
0 & 0 & 0 & 0
\end{array}\right],
$$

which was studied as in Example 2.14. $\mathbb{A}_{2}$ is symmetric, so the numerical results can be obtained as follows.

\begin{tabular}{|c|c|c|c|}
\hline$m$ & $\rho\left(\mathbb{A}_{m-1}\right)^{\frac{1}{m}}$ & $\rho\left(\mathbb{T}_{m}\right)^{\frac{1}{m}}$ & $\rho\left(\mathbb{A}_{m}\right)^{\frac{1}{m}}$ \\
\hline 2 & 1.3415037626 & 1.5537739740 & 1.5537739740 \\
3 & 1.3804413572 & 1.4892228485 & 1.5370592754 \\
4 & 1.4041128626 & 1.5069022259 & 1.5284545258 \\
5 & 1.4201397131 & 1.5017251916 & 1.5233415461 \\
6 & 1.4316975290 & 1.5035148094 & 1.5199401525 \\
7 & 1.4404277508 & 1.5028716910 & 1.5175154443 \\
8 & 1.4472546963 & 1.5031163748 & 1.5156994341 \\
9 & 1.4527395436 & 1.5030208210 & 1.5142884861 \\
10 & 1.4572426033 & 1.5030591603 & 1.5131606734 \\
11 & 1.4610058138 & 1.5030435026 & 1.5122385423 \\
12 & 1.4641976583 & 1.5030500001 & 1.5114705290 \\
13 & 1.4669390746 & 1.5030472703 & 1.5108209763 \\
14 & 1.4693191202 & 1.5030484295 & 1.5102644390 \\
15 & 1.4714048275 & 1.5030479329 & 1.5097822725 \\
16 & 1.4732476160 & 1.5030481473 & 1.5093605030 \\
\hline
\end{tabular}

Notably, both $\rho\left(\mathbb{A}_{m}\right)^{\frac{1}{m}}$ and $\rho\left(\mathbb{T}_{2 m}\right)^{\frac{1}{2 m}}$ are monotonically decreasing in $m$. In contrast, $\rho\left(\mathbb{A}_{m-1}\right)^{\frac{1}{m}}$ and $\rho\left(\mathbb{T}_{2 m+1}\right)^{\frac{1}{2 m+1}}$ are monotonically increasing in $m$, that $\rho\left(\mathbb{T}_{2 m}\right)^{\frac{1}{2 m}}$ gives better upper bound than $\rho\left(\mathbb{A}_{m}\right)^{\frac{1}{m}}$. That $\rho\left(\mathbb{T}_{2 m+1}\right)^{\frac{1}{2 m+1}}$ are lower bounds is conjectured. If they were, then $\rho\left(\mathbb{T}_{m}\right)^{\frac{1}{m}}$ would yield a very sharp estimates.

4. More symbols on larger lattice. As mentioned in the introduction, many physical and engineering problems involve many (more than two) symbols and larger lattices. Therefore, the results found in the previous sections must be extended to any finite number of symbols $p \geq 2$ on any finite square lattice $\mathbb{Z}_{2 l \times 2 l, l \geq 1}$. The results are only outlined here, and the details are left to the readers. Proofs of theorems are omitted for brevity.

For fixed $p \geq 2$ and $l \geq 1$, denote by

$$
q=p^{l^{2}} \text {. }
$$

The horizontal and vertical transition matrices are given by

$$
\mathbb{A}_{2}=\left[\begin{array}{cccc}
a_{1,1} & a_{1,2} & \cdots & a_{1, q^{2}} \\
a_{2,1} & a_{2,2} & \cdots & a_{2, q^{2}} \\
\vdots & \vdots & \ddots & \vdots \\
a_{q^{2}, 1} & a_{q^{2}, 2} & \cdots & a_{q^{2}, q^{2}}
\end{array}\right]
$$


and

$$
\mathbb{B}_{2}=\left[\begin{array}{cccc}
b_{1,1} & b_{1,2} & \cdots & b_{1, q^{2}} \\
b_{2,1} & b_{2,2} & \cdots & b_{2, q^{2}} \\
\vdots & \vdots & \ddots & \vdots \\
b_{q^{2}, 1} & b_{q^{2}, 2} & \cdots & b_{q^{2}, q^{2}}
\end{array}\right]
$$

respectively.

Now, $\mathbb{A}_{2}$ and $\mathbb{B}_{2}$ are related to each other by

$$
\mathbb{A}_{2}=\left[\begin{array}{cccc}
A_{2 ; 1} & A_{2 ; 2} & \cdots & A_{2 ; q} \\
A_{2 ; q+1} & A_{2 ; q+2} & \cdots & A_{2 ; 2 q} \\
\vdots & \vdots & \ddots & \vdots \\
A_{2 ; q(q-1)+1} & \cdots & \cdots & A_{2 ; q^{2}}
\end{array}\right]
$$

where

$$
A_{2 ; \alpha}=\left[\begin{array}{cccc}
b_{\alpha, 1} & b_{\alpha, 2} & \cdots & b_{\alpha, q} \\
b_{\alpha, q+1} & b_{\alpha, q+2} & \cdots & b_{\alpha, 2 q} \\
\vdots & \vdots & \ddots & \vdots \\
b_{\alpha, q(q-1)+1} & b_{\alpha, q(q-1)+2} & \cdots & b_{\alpha, q^{2}}
\end{array}\right]
$$

and

$$
\mathbb{B}_{2}=\left[\begin{array}{cccc}
B_{2 ; 1} & B_{2 ; 2} & \cdots & B_{2 ; q} \\
B_{2 ; q+1} & B_{2 ; q+2} & \cdots & B_{2 ; 2 q} \\
\vdots & \vdots & \ddots & \vdots \\
B_{2 ; q(q-1)+1} & \cdots & \cdots & B_{2 ; q^{2}}
\end{array}\right]
$$

where

$$
B_{2 ; \alpha}=\left[\begin{array}{cccc}
a_{\alpha, 1} & a_{\alpha, 2} & \cdots & a_{\alpha, q} \\
a_{\alpha, q+1} & a_{\alpha, q+2} & \cdots & a_{\alpha, 2 q} \\
\vdots & \vdots & \ddots & \vdots \\
a_{\alpha, q(q-1)+1} & a_{\alpha, q(q-1)+2} & \cdots & a_{\alpha, q^{2}}
\end{array}\right],
$$

respectively, where $1 \leq \alpha \leq q^{2}$. The column matrices $\widetilde{\mathbb{A}_{2}}$ and $\widetilde{\mathbb{B}_{2}}, \mathbb{A}_{2}$ and $\mathbb{B}_{2}$ are defined as in (2.1) and (2.2). For higher order transition matrices $\mathbb{A}_{n}, n \geq 3$, are defined as

$$
\mathbb{A}_{n}=\left[\begin{array}{cccc}
A_{n ; 1} & A_{n ; 2} & \cdots & A_{n ; q} \\
A_{n ; q+1} & A_{n ; q+2} & \cdots & A_{n ; 2 q} \\
\vdots & \vdots & \ddots & \vdots \\
A_{n ; q(q-1)+1} & A_{n ;(q-1) q+2} & \cdots & A_{n ; q^{2}}
\end{array}\right]
$$

where

$$
\mathbb{A}_{n ; \alpha}=\left[\begin{array}{llll}
b_{\alpha, 1} A_{n-1 ; 1} & b_{\alpha, 2} A_{n-1 ; 2} & \cdots & b_{\alpha, q} A_{n-1 ; q} \\
b_{\alpha, q+1} A_{n-1 ; q+1} & b_{\alpha, q+2} A_{n-1 ; q+2} & \cdots & b_{\alpha, 2 q} A_{n-1 ; 2 q} \\
\vdots & \vdots & \ddots & \vdots \\
b_{\alpha, q(q-1)+1} A_{n-1 ; q(q-1)+1} & b_{\alpha, q(q-1)+2} A_{n-1 ; q(q-1)+2} & \cdots & b_{\alpha, q^{2}} A_{n ; q^{2}}
\end{array}\right] .
$$


Rewriting the indices of $A_{n ; \alpha}$ as follows, facilitates matrix multiplication.

$$
\mathbb{A}_{n}=\left[\begin{array}{cccc}
A_{n ; 11} & A_{n ; 12} & \cdots & A_{n ; 1 q} \\
A_{n ; 21} & A_{n ; 22} & \cdots & A_{n ; 2 q} \\
\vdots & \vdots & \ddots & \vdots \\
A_{n ; q 1} & A_{n ; q 2} & \cdots & A_{n ; q q}
\end{array}\right]
$$

Clearly, $A_{n ; \alpha}=A_{n ; j_{1} j_{2}}$, where

$$
\alpha=\alpha\left(j_{1}, j_{2}\right)=q\left(j_{1}-1\right)+j_{2} .
$$

For $m \geq 2$, the elementary pattern in the entries of $\mathbb{A}_{n}^{m}$ is given by

$$
A_{n ; j_{1} j_{2}} A_{n ; j_{2} j_{3}} \cdots A_{n ; j_{m} j_{m+1}}
$$

where $j_{s} \in\{1,2, \cdots, q\}$.

The lexicographic order for multiple indices

$$
J_{m+1}=\left(j_{1} j_{2} \cdots j_{m} j_{m+1}\right)
$$

is introduced by

$$
\chi\left(J_{m+1}\right)=1+\sum_{l=2}^{m} q^{m-l}\left(j_{l}-1\right) .
$$

Specify

$$
A_{m, n ; \alpha}^{(k)}=A_{n ; j_{1} j_{2}} A_{n ; j_{2} j_{3}} \cdots A_{n ; j_{m} j_{m+1}},
$$

where $\alpha=\alpha\left(j_{1}, j_{m+1}\right)$ satisfies (4.11) and $k=\chi\left(J_{m+1}\right)$ is as given in (4.12). Based on this arrangement, $\mathbb{A}_{n}^{m}$ can be written as

$$
\mathbb{A}_{n}^{m}=\left[\begin{array}{cccc}
A_{m, n ; 1} & A_{m, n ; 2} & \cdots & A_{m, n ; q} \\
A_{m, n ; q+1} & A_{m, n ; q+2} & \cdots & A_{m, n ; 2 q} \\
\vdots & \vdots & \ddots & \vdots \\
A_{m, n ; q(q-1)+1} & A_{m, n ; q(q-1)+2} & \cdots & A_{m, n ; q^{2}}
\end{array}\right]
$$

where

$$
A_{m, n ; \alpha}=\sum_{k=1}^{q^{m-1}} A_{m, n ; \alpha}^{(k)} .
$$

Moreover, $X_{m, n ; \alpha}=\left(A_{m, n ; \alpha}^{(k)}\right)^{t}$, where $1 \leq k \leq q^{m-1}$ and $X_{m, n ; \alpha}$ is a $q^{m-1}$-vector that comprise all elementary patterns in $A_{m, n ; \alpha}$. The ordering matrix $\mathbb{X}_{m, n}$ of $\mathbb{A}_{n}^{m}$ is now defined as

$$
\mathbb{X}_{m, n}=\left[\begin{array}{cccc}
X_{m, n ; 1} & X_{m, n ; 2} & \cdots & X_{m, n ; q} \\
X_{m, n ; q+1} & X_{m, n ; q+2} & \cdots & X_{m, n ; 2 q} \\
\vdots & \vdots & \ddots & \vdots \\
X_{m, n ; q(q-1)+1} & X_{m, n ; q(q-1)+2} & \cdots & X_{m, n ; q^{2}}
\end{array}\right],
$$


and $X_{m, n+1 ; \beta}$ can be reduced to $X_{2, n ; \beta}$ by multiplication with connecting matrices $C_{m ; \alpha, \beta}$. The connecting operator $\mathbb{C}_{m}$ is defined as follows.

Definition 4.1. For $m \geq 2$, define

$$
\mathbb{C}_{m}=\left[\begin{array}{cccc}
C_{m ; 1,1} & C_{m ; 1,2} & \cdots & C_{m ; 1, q^{2}} \\
C_{m ; 2,1} & C_{m ; 2,2} & \cdots & C_{m ; 2, q^{2}} \\
\vdots & \vdots & \ddots & \vdots \\
C_{m ; q^{2}, 1} & C_{m ; q^{2}, 2} & \cdots & C_{m ; q^{2}, q^{2}}
\end{array}\right]
$$

$=\left[\begin{array}{ccc|c|ccc}S_{m ; 1,1} & \cdots & S_{m ; 1, q} & & S_{m ; q, 1} & \cdots & S_{m ; q, q} \\ \vdots & \ddots & \vdots & \cdots & \vdots & \ddots & \vdots \\ S_{m ; 1, q(q-1)+1} & \cdots & S_{m ; 1, q^{2}} & & S_{m ; q, q(q-1)+1} & \cdots & S_{m ; q, q^{2}} \\ \hline & \vdots & & \ddots & & \vdots & \\ \hline S_{m ; q(q-1)+1,1} & \cdots & S_{m ; q(q-1)+1, q} & & S_{m ; q^{2}, 1} & \cdots & S_{m ; q^{2}, q} \\ \vdots & \ddots & \vdots & \cdots & \vdots & \ddots & \vdots \\ S_{m ; q(q-1)+1, q(q-1)+1} & \cdots & S_{m ; q(q-1)+1, q^{2}} & & S_{m ; q^{2}, q(q-1)+1} & \cdots & S_{m ; q^{2}, q^{2}}\end{array}\right]$

where

(4.14)

$$
C_{m ; \alpha, \beta}=\left(\left(B_{2 ; \alpha}\right)_{q \times q} \circ\left(\hat{\otimes} \mathbb{B}_{2}^{m-2}\right)_{q \times q}\right)_{q^{m-1} \times q^{m-1}} \circ\left(E_{q^{m-2} \times q^{m-2}} \otimes \tilde{A}_{2 ; \beta}\right)_{q^{m-1} \times q^{m-1}} .
$$

Like Theorem 2.4, $C_{m+1 ; \alpha, \beta}$ can be obtained in terms of $C_{m ; \gamma, \beta}$.

Theorem 4.2. For any $m \geq 2$ and $1 \leq \alpha, \beta \leq q^{2}$

$$
C_{m+1 ; \alpha, \beta}=\left[\begin{array}{cccc}
a_{\alpha ; 1} C_{m ; 1, \beta} & a_{\alpha ; 2} C_{m ; 2, \beta} & \cdots & a_{\alpha ; q} C_{m ; q, \beta} \\
a_{\alpha ; q+1} C_{m ; q+1, \beta} & a_{\alpha ; q+2} C_{m ; q+2, \beta} & \cdots & a_{\alpha ; 2 q} C_{m ; 2 q, \beta} \\
\vdots & \vdots & \ddots & \vdots \\
a_{\alpha ; q(q-1)+1} C_{m ; q(q-1)+1, \beta} & a_{\alpha ; q(q-1)+2} C_{m ; q(q-1)+2, \beta} & \cdots & a_{\alpha ; q^{2}} C_{m ; q^{2}, \beta}
\end{array}\right] .
$$

Denote by

$$
A_{m, n+1 ; \alpha}^{(k)}=\left[\begin{array}{cccc}
A_{m, n+1 ; \alpha ; 1}^{(k)} & A_{m, n+1 ; \alpha ; 2}^{(k)} & \cdots & A_{m, n+1 ; \alpha ; q}^{(k)} \\
A_{m, n+1 ; \alpha ; q+1}^{(k)} & A_{m, n+1 ; \alpha ; q+2}^{(k)} & \cdots & A_{m, n+1 ; \alpha ; 2 q}^{(k)} \\
\vdots & \vdots & \ddots & \vdots \\
A_{m, n+1 ; \alpha ; q(q-1)+1}^{(k)} & A_{m, n+1 ; \alpha ; q(q-1)+2}^{(k)} & \cdots & A_{m, n+1 ; \alpha ; q^{2}}^{(k)}
\end{array}\right]
$$

and $X_{m, n+1 ; \alpha ; \beta}=\left(A_{m, n+1 ; \alpha ; \beta}^{(k)}\right)^{t}$ where $A_{m, n+1 ; \alpha ; \beta}^{(k)}$ is a linear combination of $A_{m, n ; \gamma}^{(l)}$. Now, Theorem 2.5 can be generalized to the following theorem.

Theorem 4.3. For any $m \geq 2$ and $n \geq 2$, let $S_{m ; \alpha, \beta}$ be as given in (4.13) and (4.14). Then $X_{m, n+1 ; \alpha ; \beta}=S_{m ; \alpha, \beta} X_{m, n ; \beta}$. 


\section{REFERENCES}

[1] J.C. BAn, K.P. Chien AND S.S. Lin, Spatial disorder of CNN-with asymmetric output funtion, International J. of Bifurcation and Chaos, 11 (2001), pp. 2085-2095.

[2] J.C. BAn, C.H. Hsu AND S.S. Lin, Spatial disorder of cellular neural network-with biased term, International J. of Bifurcation and Chaos, 12 (2002), pp. 525-534.

[3] J.C. BAN, S.S. Lin AND C.W. SHIH, Exact number of mosaic patterns in one-dimensional cellular neural networks, International J. of Bifurcation and Chaos, 11 (2001), pp. 16451653.

[4] J.C. BAN AND S.S. Lin, Patterns generation and transition matrices in multi-dimensional lattice models, Discrete Contin. Dyn. Syst., 13:3 (2005), pp. 637-658.

[5] J.C. BAN, S.S. Lin AND Y.H. Lin, Patterns generation and spatial entropy in three-dimensional lattice models, preprint (2005).

[6] J.C. Ban, S.S. Lin AND Y.H. Lin, Primitivity of subshifts of finite type in two-dimensional lattice models, preprint (2005).

[7] P.W. Bates and A. Chmaj, A discrete convolution model for phase transitions, Arch. Rat. Mech. Anal, 150 (1999), pp. 281-305.

[8] P.W. Bates, K. Lu And B. WANG, Attractors for lattice dynamical systems, International J. of Bifurcation and Chaos, 11 (2001), pp. 143-153.

[9] J. BeLl, Some threshold results for modes of myelinated nerves, Math. Biosci., 54 (1981), pp. 181-190.

[10] J. Bell AND C. Cosner, Threshold behavior and propagation for nonlinear differentialdifference systems motivated by modeling myelinated axons, Quart. Appl. Math., 42 (1984), pp. 1-14.

[11] R. J. Baxter, Eight-vertex model in lattice statistics., Phys. Rev. Lett., 26 (1971), pp. 832-833.

[12] J.W. CAHN, Theory of crystal growth and interface motion in crystalline materials, Acta Metallurgica, 8 (1960), pp. 554-562.

[13] S.N. Chow And J. Mallet-Paret, Pattern formation and spatial chaos in lattice dynamical systems II, IEEE Trans. Circuits Systems, 42 (1995), pp. 752-756.

[14] S.N. Chow, J. Mallet-Paret and E. S. Van Vleck, Dynamics of lattice differential equations, International J. of Bifurcation and Chaos, 6 (1996), pp. 1605-1621.

[15] S.N. Chow, J. Mallet-Paret and E.S. Van Vleck, Pattern formation and spatial chaos in spatially discrete evolution equations, Random Comput. Dynam., 4 (1996), pp. 109-178.

[16] L.O. ChuA, CNN: A paradigm for complexity, World Scientific Series on Nonlinear Science, Series A 31. World Scietific, Singapore, 1998.

[17] L.O. Chua, K.R. Crounse, M. Hasler and P. Thiran, Pattern formation properties of autonomous cellular neural networks, IEEE Trans. Circuits Systems, 42 (1995), pp. 757774 .

[18] L.O. Chua and T. Roska, The CNN paradigm, IEEE Trans. Circuits Systems, 40 (1993), pp. $147-156$.

[19] L.O. Chua And L. Yang, Cellular neural networks: Theory, IEEE Trans. Circuits Systems, 35 (1988), pp. 1257-1272.

[20] L.O. Chua And L. YAng, Cellular neural networks: Applications, IEEE Trans. Circuits Systems, 35 (1988), pp. 1273-1290.

[21] G.B. Ermentrout, Stable periodic solutions to discrete and continuum arrays of weakly coupled nonlinear oscillators, SIAM J. Appl. Math., 52 (1992), pp. 1665-1687.

[22] G.B. Ermentrout And N. Kopell, Inhibition-produced patterning in chains of coupled nonlinear oscillators, SIAM J. Appl. Math., 54 (1994), pp. 478-507.

[23] G.B. Ermentrout, N. Kopell and T. L. Williams, On chains of oscillators forced at one end, SIAM J. Appl. Math., 51 (1991), pp. 1397-1417.

[24] T. Eveneux and J.P. Laplante, Propagation failure in arrays of coupled bistable chemical reactors, J. Phys. Chem., 96 (1992), pp. 4931-4934.

[25] W.J. Firth, Optical memory and spatial chaos, Phys. Rev. Lett. 61 (1988), pp. 329-332.

[26] F.R. Gantmacher, The theory of matrices, 2 vols. Chelsea, New York, 1959.

[27] R.A. Horn and C.R. Johnson, Matrix analysis, Cambridge University Press, Cambridge, 1990.

[28] C.H. Hsu, J. JuAng, S.S. Lin, AND W.W. Lin, Cellular neural networks: local patterns for general template, International J. of Bifurcation and Chaos, 10 (2000), pp. 1645-1659.

[29] J. JuAng And S.S. Lin, Cellular Neural Networks: Mosaic pattern and spatial chaos, SIAM J. Appl. Math., 60 (2000), pp. 891-915.

[30] J. JuAng, S.S. Lin, W.W. Lin And S.F. ShieH, The spatial entropy of two-dimensional subshifts of finite type, International J. of Bifurcation and Chaos, 10 (2000), pp. 2845-2852. 
[31] J.P. KeEnER, Propagation and its failure in coupled systems of discrete excitable cells, SIAM J. Appl. Math., 47 (1987), pp. 556-572.

[32] J.P. KEENER, The effects of discrete gap junction coupling on propagation in myocardium, J. Theor. Biol., 148 (1991), pp. 49-82.

[33] A.L. Kimball, A. Varghese and R.L. Winslow, Simulating cardiac sinus and atrial netwok dynamics on the connection machine, Phys. D, 64 (1993), pp. 281-298.

[34] E.H. LiEB, Exact solution of the problem of the entropy of two-dimensional ice, Phys. Rev. Lett., 18 (1967), pp. 692-694.

[35] E.H. LiEB, Exact solution of the $f$ model of an antiferroelectric, Phys. Rev. Lett., 18 (1967), pp. 1046-1048.

[36] E.H. Lieb, Exact Solution of the Two-Dimensional Slater KDP Model of a Ferroelectric, Phys. Rev. Lett., 19 (1967), pp. 108-110.

[37] E.H. LieB, The Residual Entropy of Square Ice, Phys. Rev., 162 (1967), pp. 162-172.

[38] E.H. LiEB, Ice, ferro- and antiferroelectrics, in methods and problems in theoretical physics, in honour of R.E. Peierls, proceedings of the 1967 Birmingham conference, (North-Holland, 1970) pp. 21-28.

[39] S.S. Lin AND T.S. YANG, Spatial entropy of one-dimensional celluar neural network, International J. of Bifurcation and Chaos, 10 (2000), pp. 2129-2140.

[40] S.S. Lin AND T.S. YANG, On the spatial entropy and patterns of two-dimensional cellular neural networks, International J. of Bifurcation and Chaos, 12 (2002), pp. 115-128.

[41] D. Lind and B. Marcus, An introduction to symbolic dynamics and coding, Cambridge University Press, Cambridge, (1995).

[42] A. Lindenmayer and P. Prusinkiewicz, The algorithmic beauty of plants, Springer-Verlag, New York, (1990).

[43] N.G. Markley and M.E. Paul, Maximal measures and entropy for $Z^{\nu}$ subshifts of finite type, Classical mechanics and dynamical systems, Medford, Mass., (1979) pp. 135-157, Lecture Notes in Pure and Appl. Math., 70, Dekker, New York, (1981).

[44] N.G. Markley and M.E. Paul, Matrix subshifts for $Z^{\nu}$ symbolic dynamics, Proc. London Math. Soc., (3) 43 (1981), pp. 251-272.

[45] L. Onsager, Crystal statistics. I. A two-dimensional model with an order-disorder transition., Phys. Rev., 65 (1944), pp. 117-149.

[46] C.N. YANG AND C.P. YANG, One-dimensional chain of anisotropic spin-spin interactions. I. Proof of Bethe's hypothesis for ground state in a finite system., Phys. Rev., 150 (1966a), pp. 321-327.

[47] C.N. YAng And C.P. YANG, One-dimensional chain of anisotropic spin-spin interactions. II. Properties of the ground-state energy per lattice site for an infinite system., Phys. Rev., 150 (1966b), pp. 327-339.

[48] C.N. YANG AND C.P. YANG, One-dimensional chain of anisotropic spin-spin interactions. III. Applications., Phys. Rev., 151 (1966c), pp. 258-264. 